\title{
Homoclinic tangencies near cascades of period doubling bifurcations
}

by

\author{
Eleonora CATSIGERAS \\ IMERL, Facultad de Ingeniería C.C. 30, Montevideo, Unuguay. \\ E-mail: eleonora@fing.edu.uy \\ and

\section{Heber ENRICH *} \\ IMERL, Facultad de Ingeniería C.C. 30, Montevideo, Uruguay. \\ E-mail: enrich@fing.edu.uy
}

\begin{abstract}
We consider perturbations of the Feigenbaum map in $n$ dimensions. In the analytic topology we prove that the maps that are accumulated by period doubling bifurcations are approximable with homoclinic tangencies. We also develop a $n$-dimensional Feigenbaum theory in the $C^{r}$ topology, for $r$ large enough. We apply this theory to extend the result of approximation with homoclinic tangencies for $C^{r}$ maps.

(C) 1998 L'Association Publications de l'Institut Henri Poincaré. Published by Elsevier B.V. All rights reserved
\end{abstract}

RÉSUMÉ. - On considère des perturbations de la transformation de Feigenbaum en dimension $n$. Dans la topologie analytique on prouve que les transformations qui sont accumulées par des bifurcations de duplication de période sont approchées par des tangences homocliniques. On développe aussi une théorie de Feigenbaum $n$-dimensionelle dans la topologie $C^{r}$, avec $r$ suffisamment grand. Cette théorie est appliquée pour étendre le résultat d'approximation par tangences homocliniques pour les transformations $C^{r}$.

(C) 1998 L'Association Publications de l'Institut Henri Poincaré. Published by Elsevier B.V. All rights reserved

* Both authors were partially supported by IMPA-CNPq, Brazil. 


\section{INTRODUCTION}

The one-parameter unfolding of a homoclinic tangency, for locally dissipative maps of class $C^{r}(r \geq 3)$, in dimension two, originates important dynamical phenomena [13] [15] [19] [16]. For instance: horseshoes and hyperbolic sets, cascades of period doubling bifurcations [21], maps with infinitely many sinks [12], Hénon-like attractors [11], [1]. Some of the results are also valid in higher dimensions [17], [20], [18], [10]. In other words, the families unfolding a homoclinic tangency have many of the known global bifurcations. They are notable examples of global unstable systems. It is not known if the homoclinic bifurcations are in general necessary for global unstability. Precisely, J. Palis has formulated the following:

CONJECTURE [16]. - The subset $\mathcal{H}$ of diffeomorphisms that are either hyperbolic (i.e. with hyperbolic limit set and no cycles) or homoclinic bifurcating is dense in the space of $C^{\infty}$ surface diffeomorphisms.

When formulating the question, J. Palis has also presented the following program: try to approximate with homoclinic hifurcations some particular global unstabilities, as for example:

1. diffeomorphisms having an attractor (as discovered by Feigenbaum and independently by Coullet and Tresser [6], [3]), at the accumulation of period doubling bifurcations.

2. diffeomorphisms having a Hénon-like attractor.

3. diffeomorphisms exhibiting infinitely many coexisting sinks.

To address the first case of the program above, we will consider a renormalization $T$ of $n$-dimensional perturbations of the Feigenbaum map $\Phi$.

The spectral properties of $d T(\Phi)$ in $n$ dimensions were studied by Collet, Eckmann and Koch [2] in the analytic topology. They proved that $\Phi$ is a hyperbolic fixed point of the renormalization $T$ with a single unstable direction and that the maps in the stable manifold are accumulated by period doubling bifurcations.

For one-dimensional analytic maps in a neighborhood of the Feigenbaum map, the accumulation of the period doubling bifurcations are approximated by band-merging maps. This result was proved by Eckmann and Wittwer [5].

Applying these results we can provide a partial answer to the part 1 of the program above: $n$-dimensional analytic maps in the stable manifold 
of $\Phi$ are the accumulation of period doubling bifurcations and can be approximated with homoclinic tangencies.

A. M. Davie ([4]) has developed an one-dimensional Feigenbaum theory in the $C^{2+\theta}$ topology. He proves that, although the renormalization is not Fréchet differentiable in that space, the Feigenbaum map is a topologically hyperbolic fixed point. He shows the existence of a $C^{1}$ stable submanifold of codimension one in the space $C^{2+\theta}$.

Following the ideas of A. M. Davie we develop a $n$-dimensional Feigenbaum theory in the $C^{r}$ topology, for $r$ large enough. Some of the arguments of [4] have to be modified to work in a $n$-dimensional setting. That is why we present a detailed exposition of the theory that occupies entirely the sections 2 and 3 . We conclude that there is a topologically hyperbolic behaviour of the renormalization near $\Phi$ in the space of $n$-dimensional $C^{r}$ maps, and the existence of the stable submanifold.

Applying these results we can improve the first answer to the question of approximation with homoclinic tangencies: also $n$-dimensional $C^{r}$ maps in the stable manifold of $\Phi$ are cascades of period doubling bifurcations which are approximable with homoclinic tangencies.

\section{THE MAIN THEOREMS}

We will denote $\mathcal{H}_{D}$ the space of bounded real analytic maps, defined in a neighborhood $D$ of $[-1,1] \times\{\mathbf{0}\}$ in $\mathbf{C}^{n}$ to $\mathbf{C}^{n}$. The map $\Phi$, as will be defined in 1.2 , is a particular endomorphism of $\mathcal{H}_{D}$, transforming $D$ into an one-dimensional image, and will be called the Feigenbaum map in $n$ dimensions.

THEOREM 1. - In the space $\mathcal{H}_{D}$ there exists a codimension one manifold $\mathcal{W}$, containing the Feigenbaum map $\Phi$, such that any differentiable curve $\left\{G_{\mu}\right\}$ in $\mathcal{H}_{D}$ that intersects transversally $\mathcal{W}$ at $G_{0}$ verifies:

a) It has a sequence of period doubling bifurcations for parameter values $\mu_{m} \rightarrow 0$ monotonely.

b) There exists $\bar{\mu}_{m} \rightarrow 0$ (monotonely, at the other side of 0 than $\mu_{m}$ ), such that $G_{\bar{\mu}_{m}}$ exhibits a homoclinic tangency.

The part $a$ ) of the theorem above appears in [2].

We denote $C^{r}$ the set of $C^{r}$ maps defined in a neighborhood $D$ of $[-1,1] \times\{\mathbf{0}\} \subset \mathbf{R}^{\mathbf{n}}$ to $\mathbf{R}^{n}$. 
THEOREM 2. - For $r$ large enough there exists in the space $C^{r}$ a codimension one manifold $\mathcal{W}$, containing the Feigenbaum map $\Phi$, such that any differentiable curve $\left\{G_{\mu}\right\}$ in $C^{r}$ that intersects transversally $\mathcal{W}$ at $G_{0}$ verifies:

a) It has a sequence of period doubling bifurcations for parameter values $\mu_{m} \rightarrow 0$ monotonely.

b) There exists $\bar{\mu}_{m} \rightarrow 0$ (monotonely, at the other side of 0 than $\mu_{m}$ ), such that $G_{\bar{\mu}_{m}}$ exhibits a homoclinic tangency.

In the section 1 we prove the first theorem, and in the sections 2 and 3 the second one. The first theorem has been proved by the first author alone. The second theorem has been proved jointly.

We thank J. Palis for posing the problem, as well as for motivating conversations and constant support, and $\mathrm{K}$. Khanin for very useful discussions and suggestions. We also thank W. de Melo, O. Lanford, and A. M. Davie. Finally, we thank to IMPA, Rio de Janeiro, for its hospitality.

\section{THE ANALYTIC PERTURBATIONS OF THE FEIGENBAUM MAP}

In this section we develop the theory in the analytic case, using the fact that the renormalization is differentiable with derivative that is a compact operator whose spectrum is computable. In the subsection 1.1 we analyse the spectrum of the renormalization and prove the part $a$ ) of the theorem 1. In the subsection 1.2 we find the homoclinic tangencies and prove the part $b$ ).

\subsection{Spectral analysis of the renormalization}

Let us state some results in dimension one that give an understanding of the cascades of period doubling bifurcations.

Let $\tilde{D}$ be a neighborhood of $[-1,1]$ in $\mathbf{C}$, and $\mathcal{H}_{\tilde{D}}$ the space of real analytic maps defined and bounded in $\tilde{D}$. It is a Banach space with the supremum norm. In $\mathcal{H}_{\tilde{D}}$ let $\hat{M}$ be the manifold

$\hat{M}=\left\{\psi \in \mathcal{H}_{\tilde{D}}: \psi(z)=g\left(z^{2}\right)\right.$ for some $g$ real analytic, $\left.g^{\prime} \neq 0, g(0)=1\right\}$

The renormalization transformation $\hat{\mathcal{F}}$ is defined as:

$$
(\hat{\mathcal{F}} \psi)(x)=\psi(1)^{-1} \psi \circ \psi(\psi(1) x)
$$

applied to the maps $\psi \in \hat{M}$ such that $-1<\psi(1)<0$ and $\psi(\psi(1) \tilde{D}) \subset \tilde{D}$. 
The following theorem provides some properties of $\hat{\mathcal{F}}$ :

THEOREM 1.1. - If the neighborhood $\tilde{D}$ is small enough, then:

a) There exists $\varphi \in \hat{M}$ fixed by $\hat{\mathcal{F}}$. The Schwarzian derivative $S \varphi$ is negative. Moreover $\varphi^{2}(0)=\varphi(1)=\lambda=-.3995 \ldots$ and $\varphi^{\prime}(1)=\lambda^{-1}$

b) $\hat{\mathcal{F}}$ is a $C^{\infty}$ transformation, and $d \hat{\mathcal{F}}(\varphi)$ is a compact operator having a single eigenvalue $\delta=4.6692 \ldots$ of modulus greater or equal than 1 , which is simple.

c) The unstable manifold $\hat{\mathcal{W}}^{u}(\varphi) \subset \hat{M}$ intersects transversally the codimension one manifold $\hat{\Sigma}_{1}$ of period doubling bifurcations, defined as follows:

$$
\hat{\Sigma}_{1}=\left\{\psi \in \hat{M}: \psi^{\prime}\left(x_{0}\right)=-1 \text { for } x_{0} \text { fixed by } \psi\right\}
$$

Proof. - See O. Lanford III's article [9]. This theorem was conjectured in [6], [3] and [7].

The Feigenbaum map in dimension one is the map $\varphi$ of the theorem above.

Following Collet, Eckmann and Koch [2], let us take a neighborhood $D$ in $\mathbf{C}^{n}$ of the interval $[-1,1] \times\{0\}$. Our functional space $\mathcal{H}_{D}$ will be the Banach real space formed by the real analytic maps defined and bounded in $D$ with the supremum norm.

Usually we will consider only the restrictions to $\mathbf{R}^{n}$ of the maps in $\mathcal{H}_{D}$. For simplicity we will not use a different notation to refer to the restriction.

Let us fix $\alpha \in \mathbf{R}^{n-1}, \alpha \neq 0$, and define $\theta: \mathbf{C}^{n} \mapsto \mathbf{C}$, and $\theta_{0}: \mathbf{C} \mapsto \mathbf{C}$, as follows:

$$
\begin{aligned}
\theta\left(z_{0}, Z\right) & =z_{0}^{2}-\alpha \cdot Z \\
\theta_{0}(z) & =z^{2}
\end{aligned}
$$

DEFINITION 1.2. - The Feigenbaum map in $n$ dimensions is the map:

$$
\Phi=(f \circ \theta, \mathbf{0}): D \subset \mathbf{C}^{n} \mapsto \mathbf{C}^{n}
$$

where $f \circ \theta_{0}=\varphi$ is the Feigenbaum map in dimension one.

For fixed $\alpha$, there exists $D$ small enough such that $\theta(D)$ is contained in the domain of $f$, and therefore $\Phi$ is well defined.

Being $\lambda=\varphi(1)=-.3995 \ldots$, let us define $\Lambda: \mathbf{C}^{n} \mapsto \mathbf{C}^{n}$, the linear rescaling $\Lambda\left(z_{0}, Z\right)=\left(\lambda z_{0}, \lambda^{2} Z\right)$, and a (first) renormalization transformalion:

$$
\mathcal{N} G=\Lambda^{-1} \circ G \circ G \circ \Lambda
$$

for all $G \in \mathcal{H}_{D}$ in a neighborhood of $\Phi$. 
The renormalization transformation $\mathcal{N}$ will be modified later (substituting the linear rescaling $\Lambda$ with a nonlinear change of coordinates), to get a new renormalization transformation $T$ that will have some desired properties. Observe that the Feigenbaum map $\Phi$ is fixed by $\mathcal{N}$ and $d \mathcal{N}(\Phi) u=\Lambda^{-1} \circ(u \circ \Phi+D \Phi \circ \Phi \cdot u) \circ \Lambda$.

Remark 1.3. - For any given real analytic map $\sigma: \mathbf{C}^{n} \mapsto \mathbf{C}^{n}$ we will denote

$$
\Psi_{\sigma}=-\sigma \circ \Phi+D \Phi \cdot \sigma
$$

It is a map in $\mathcal{H}_{D}$ tangent at $\Phi$ to the curve of maps:

$$
\left\{(I+t \sigma)^{-1} \circ \Phi \circ(I+t \sigma)\right\}, t \in(-\varepsilon, \varepsilon) \subset \mathbf{R}
$$

of analytic conjugates of $\Phi$ near $\Phi$.

THEOREM 1.4 (Collet, Eckmann and Koch) [2]

a) $\mathcal{N}$ is infinitely differentiable and $d \mathcal{N}(\Phi)$ is a compact operator whose eigenvalues of modulus greater or equal than $I$ are $1, \lambda^{-1}, \lambda^{-2}, \delta$.

b) Their respective spectral invariant subspaces $\bar{S}_{0}, \bar{S}_{1}, \bar{S}_{2}$, and $\bar{U}$, are eigenspaces.

Moreover, the subspace $\bar{U}$ is one-dimensional in $\mathcal{H}_{D}$. The subspaces $\bar{S}_{0}, \bar{S}_{1}$ and $\bar{S}_{2}$ are finite dimensional described as:

$$
\begin{gathered}
\bar{S}_{0}=\left\{\Psi_{\sigma}: \sigma\left(z_{0}, Z\right)=\left(a_{1} z_{0}, B_{2} z_{0}^{2}+A \cdot Z\right)\right\} \\
\bar{S}_{1}=\left\{\Psi_{\sigma}: \sigma\left(z_{0}, Z\right)=\left(a_{0}, B_{1} z_{0}\right)\right\} \\
\bar{S}_{2}=\left\{\Psi_{\sigma}: \sigma\left(z_{0}, Z\right)=\left(0, B_{0}\right)\right\}
\end{gathered}
$$

with $a_{0}$ and $a_{1}$ in $\mathbf{R} ; B_{0}, B_{1}$ and $B_{2}$ in $\mathbf{R}^{n-1}$; and $A \in$ $\mathcal{L}\left(\mathbf{R}^{n-1}, \mathbf{R}^{n-1}\right)$.

Proof. See [2].

For later purposes we need to modify the renormalization $\mathcal{N}$. Let us define the transformation $\mathcal{F}$, applied to the maps $G \in \mathcal{H}_{D}$ in a neighborhood of $\Phi$ :

$$
\mathcal{F}(G)=\Lambda_{G}^{-1} \circ G \circ G \circ \Lambda_{G}
$$

where $\Lambda_{G}\left(z_{0}, Z\right)=\left(\lambda_{G} z_{0}, \lambda_{G}^{2} Z\right)$ for

$$
\lambda_{G}=\frac{\pi_{1} \circ G^{2}(0, \mathbf{0})}{\pi_{1} \circ G(0, \mathbf{0})}
$$

where $\pi_{1}$ is the first coordinate projection. 
Note that $\lambda_{\Phi}=\lambda$ and $\mathcal{F}(\Phi)=\mathcal{N}(\Phi)=\Phi$. After some computations we find that for all $u=\left(u_{0}, U\right) \in \mathcal{H}_{D}$ :

$$
d \mathcal{F}(\Phi) u=d \mathcal{N}(\Phi) u+\gamma(u) \Psi_{\sigma_{1}},
$$

where:

$$
\gamma(u)=\left.\frac{1}{\lambda} d \lambda_{G}\right|_{G=\Phi}=\frac{u_{0}(1, \mathbf{0})}{\lambda}+u_{0}(0, \mathbf{0})\left(\frac{1}{\lambda^{2}}-1\right)-\frac{\alpha \cdot U(0, \mathbf{0})}{2 \lambda^{2}}
$$

and $\sigma_{1}\left(z_{0}, Z\right)=\left(z_{0}, 2 Z\right)$. We have:

$$
\Psi_{\sigma_{1}}\left(z_{0}, Z\right)=\left(-f\left(z_{0}^{2}-\alpha \cdot Z\right)+2 f^{\prime}\left(z_{0}^{2}-\alpha \cdot Z\right)\left(z_{0}^{2}-\alpha \cdot Z\right), \mathbf{0}\right)
$$

So $\gamma\left(\Psi_{\sigma_{1}}\right)=0$. Observe that $\Psi_{\sigma_{1}} \in \bar{S}_{0}$, thus it is fixed by $d \mathcal{N}(\Phi)$ and $d \mathcal{F}(\Phi)$. A consequence of (1) and of theorem 1.4, is the following:

PROPOSITION 1.5.

a) $\Phi$ is a fixed point of $\mathcal{F}$, and $d \mathcal{F}(\Phi)$ has the same spectrum that $d \mathcal{N}(\Phi)$.

b) The spectral invariant subspaces $S_{0}, S_{1}, S_{2}$ and $U$ corresponding respectively to the eigenvalues $1, \lambda^{-1}, \lambda^{-2}$ and $\delta$ have the same dimension that $\bar{S}_{0}, \bar{S}_{1}, \bar{S}_{2}$ and $\bar{U}$ of the theorem 1.4. Moreover $S_{0} \oplus S_{1} \oplus S_{2}=\bar{S}_{0} \oplus \bar{S}_{1} \oplus \bar{S}_{2}=\bar{S}$.

c) For any $u \in \mathcal{H}_{D}$ there exists $\sigma[u]$, the unique analytic map in $\mathbf{C}^{n}$ such that $\Psi_{\sigma[u]}=E u$, where $E$ is the spectral projection on $\bar{S}$. The transformation $u \mapsto \sigma[u]$ is linear and bounded.

Proof. - Let us denote $F=d \mathcal{F}(\Phi), N=d \mathcal{N}(\Phi)$. They are compact operators. Denote $\Sigma(F), \Sigma(N)$ their spectra. We know that $F \Psi_{\sigma_{1}}=N \Psi_{\sigma_{1}}=\Psi_{\sigma_{1}}$. Let $\mu \neq 0$. We assert that $\mu \in \Sigma(F)$ with multiplicity $m$, if and only if $\mu \in \Sigma(N)$ with the same multiplicity. In fact, take $\mu \in \Sigma(F)$, with spectral subspace $\operatorname{ker}(F-\mu)^{\nu}$ of dimension $m$. Define $V=\operatorname{ker}(F-\mu)^{\nu}+\left[\Psi_{\sigma_{1}}\right]$. It is invariant by $F$. The Jordan matrix $J$ of $F$ restricted to $V$ has $\mu$ in the diagonal repeated $m$ times (and a single 1 if $\mu \neq 1$ ). In the same basis, the linear operator $N$ restricted to $V$ has a triangular matrix with the same diagonal than $J$, (due to (1)). Then $\mu \in \Sigma(N)$ and has multiplicity at least $m$. Changing the roles of $N$ and $F$, our assertion is proved, and also for $\mu=1, \lambda^{-1}, \lambda^{-2}$ or $\delta$ :

$$
\operatorname{ker}(F-\mu)^{\nu}+\left[\Psi_{\sigma_{1}}\right]=\operatorname{ker}(N-\mu)^{\nu}+\left[\Psi_{\sigma_{1}}\right] .
$$

Now, part $b$ ) follows easily. Finally, $E u=\Psi_{\sigma}$ for some $\sigma$ in the set

$$
\begin{aligned}
\Sigma=\left\{\sigma: \mathbf{C}^{n} \mapsto \mathbf{C}^{n}\right. \text { analytic; } & \\
\sigma\left(z_{0}, Z\right) & \left.=\left(a_{0}+a_{1} z_{0}, B_{0}+B_{1} z_{0}+B_{2} z_{0}^{2}+A \cdot Z\right)\right\}
\end{aligned}
$$

due to theorem 1.4 . 
Let $Q: \Sigma \mapsto \bar{S}$ be the linear transformation between finite-dimensional spaces defined by $Q(\sigma)=\Psi_{\sigma}$ as in 1.3. It is easy to check that $Q$ is injective. Therefore $u \mapsto \sigma[u]=Q^{-1} E u$ is linear and bounded.

We are ready to define our final renormalization transformation in $n$ dimensions:

DEFINITION 1.6. - The renormalization transformation $T$ is:

$$
T(G)=(I-\sigma[\mathcal{F}(G)-\Phi])^{-1} \circ \mathcal{F}(G) \circ(I-\sigma[\mathcal{F}(G)-\Phi])
$$

applied to $G \in \mathcal{H}_{D}$ in a neighborhood of $\Phi$.

The renormalization $T$ was chosen so that it verifies the following properties:

COROLLARY 1.7 .

a) The map $\Phi$ is a fixed point of $T$.

b) $T$ is infinitely differentiable and $d T(\Phi)$ is a compact operator, having a single simple eigenvalue $\delta=4.6692 \ldots$ of modulus greater or equal than 1.

c) The unstable manifold $\mathcal{W}^{u}(\Phi)=\left\{\Phi_{t}\right\}$ is formed by the maps $\Phi_{t} \in \mathcal{H}_{D}$ of the form:

$$
\Phi_{t}\left(z_{0}, Z\right)=\left(f_{t}\left(z_{0}^{2}-\alpha \cdot Z\right), \mathbf{0}\right)
$$

where $f_{t}\left(z^{2}\right)=\varphi_{t}(z)$ are the one-dimensional maps of the unstable manifold $\left\{\varphi_{t}\right\}=\hat{\mathcal{W}}^{u}(\varphi)$ of the renormalization $\hat{\mathcal{F}}$ in dimension one (cf. theorem 1.1).

Proof. - Part a) can be easily verified.

Part b) follows from the proposition 1.5: in fact, taking derivatives in the equality of the definition 1.6, and denoting $F=d \mathcal{F}(\Phi)$, we get:

$$
d T(\Phi) u=F u+\sigma[F u] \circ \Phi-D \Phi \cdot \sigma[F u]=F u-\Psi_{\sigma[F u]}=(I-E) F u
$$

Now, all vectors of $\bar{S}$ are in the kernel of $d T(\Phi)$. Thus, the only unstable direction that remains has eigenvalue $\delta$, as wanted.

We now show part $c$ ). Let $\hat{M}$, defined at the beginning of this subsection, be the manifold of one-dimensional maps $\psi$. We will consider in $\mathcal{H}_{D}$ the submanifold

$$
M=\left\{\Psi \in \mathcal{H}_{D}: \Psi=(g \circ \theta, \mathbf{0}) \text { where } g \circ \theta_{0} \in \hat{M}\right\}
$$


(Recall that $\theta\left(z_{0}, Z\right)=z_{0}^{2}-\alpha \cdot Z$ and $\theta_{0}(z)=z^{2}$ ). Note that $\Phi \in M$ and that for all $\Psi=(g \circ \theta, \mathbf{0}) \in M$ in a neighborhood of $\Phi, \mathcal{F} \Psi$ is in $M$ and it is obtained computing $\hat{\mathcal{F}}\left(g \circ \theta_{0}\right)$.

The theorem 1.1 implies that $\mathcal{F}$ restricted to a neighborhood of $\Phi$ in $M$ has derivative at $\Phi$ whose spectrum exhibits a single eigenvalue $\delta$ of modulus greater or equal than 1 . Thus $T_{\Phi} M \subset \operatorname{ker}(E)$, and $\sigma[u]=0$ for all $u \in T_{\Phi} M$. As $M-\Phi \subset T_{\Phi} M$ by the definition $1.6 T \Psi=\mathcal{F} \Psi$ for all $\Psi$ in a neighborhood of $\Phi$ in $M$. As $\mathcal{F} \Psi$ is obtained computing $\hat{\mathcal{F}}$, the theorem 1.1 implies $c$ ).

Due to the theorem 1.1 we can take a parametrization $\left\{\varphi_{t}\right\}$ of $\hat{\mathcal{W}}^{u}(\varphi)$ such that $\varphi_{0}=\varphi, \hat{\mathcal{F}}\left(\varphi_{t}\right)=\varphi_{\delta t}$ and $\varphi_{-1}$ is the transversal intersection of $\hat{\Sigma}_{1}$ with $\left\{\varphi_{t}\right\}$.

By the part c) of the corollary 1.7 we have the correspondent parametrization $\left\{\Phi_{t}\right\}$ of $\mathcal{W}^{u}(\Phi)$.

The map $\Phi_{-1}$ has a fixed point $\left(x_{-1}, \mathbf{0}\right)$ with $x_{-1}$ fixed by $\varphi_{-1}$, and

$$
D \Phi_{-1}\left(x_{-1}, \mathbf{0}\right)=\left(\begin{array}{cc}
\varphi_{-1}^{\prime}\left(x_{-1}\right) & -\alpha \cdot f_{-1}^{\prime}\left(x_{-1}^{2}\right) \\
0 & \mathbf{0}
\end{array}\right)
$$

has eigenvalues -1 (simple) and 0 (with multiplicity $n-1$ ). So, there exists a neighborhood $N_{0}$ of $\Phi_{-1}$ in $\mathcal{H}_{D}$ such that all $G \in N_{0}$ has a fixed point $p_{G}$, continuation of $\left(x_{-1}, \mathbf{0}\right)$, and $D G(p(G))$ has a eigenvalue $\rho(G)$ near -1 and $n-1$ eigenvalues near 0 . In particular, for $\Phi_{t} \in N_{0}$, $p\left(\Phi_{t}\right)=\left(x_{t}, 0\right)$ and $\rho\left(\Phi_{t}\right)=\varphi_{t}^{\prime}\left(x_{t}\right)$.

Let us define

$$
\Sigma_{1}=\left\{G \in N_{0}: \rho(G)=-1\right\}
$$

It is a submanifold of codimension one in $\mathcal{H}_{D}$. Any differentiable curve of analytic maps intersecting transversally $\Sigma_{1}$ exhibits a period doubling bifurcation of period 1 to period 2 .

By the part $c$ ) of the theorem 1.1: $\left.\frac{d}{d t} \varphi_{t}^{\prime}\left(x_{t}\right)\right|_{t=-1} \neq 0$.

So $\left.\frac{d}{d t} \rho\left(\Phi_{t}\right)\right|_{t=-1} \neq 0$ and $\left\{\Phi_{t}\right\}$ intersects transversally $\Sigma_{1}$ at $t=-1$.

Remark 1.8. - As $T^{m}\left(\Phi_{-\delta^{-m}}\right)=\Phi_{-1}$, arguing as above the submanifolds $\Sigma_{m}$ of codimension one in $\mathcal{H}_{D}$, where the period doubling bifurcations of period $2^{m}$ to period $2^{m+1}$ occur, are transversal to $\left\{\Phi_{t}\right\}$ in $\Phi_{-\delta^{-m}}$.

We are ready to prove the first part of the theorem 1 .

Proof of part a) of theorem 1. - Let $\mathcal{W}=\mathcal{W}^{s}(\Phi)$. Given a differentiable curve $\left\{G_{\mu}\right\}$ transversal at $\mu=0$ to $\mathcal{W}$ its images by the renormalization Vol. 15, n ${ }^{\circ} 3-1998$. 
$T^{m}$ accumulate at $\mathcal{W}^{u}(\Phi)$ when $m \rightarrow \infty$, due to the inclination lemma ([14]). So, for $m$ sufficiently large there exists $\mu_{m}$ such that $\left\{T^{m} G_{\mu}\right\}$ intersects transversally $\Sigma_{1}$ at $\mu=\mu_{m}$. Therefore $\left\{G_{\mu}\right\}$ exhibits a period doubling bifurcation of period $2^{m}$ to $2^{m+1}$. The argument above works for any subarc of $\left\{G_{\mu}\right\}$ as near as wanted from $G_{0}$. Thus $\mu_{m} \rightarrow 0$.

\subsection{Homoclinic bifurcating maps}

Let $\left\{G_{\mu}\right\}, \mu \in[a, b]$ be a continuous arc of maps in $\mathcal{H}_{D}$. Let us suppose that for all $\mu \in[a, b]$ there exists a hyperbolic periodic point $p_{\mu}$, depending continously on $\mu$, of stable codimension one.

Let us denote $A_{\mu}^{u}$ and $A_{\mu}^{s} \quad$ compact parts of $W^{u}\left(p_{\mu}\right)$ and $W^{s}\left(p_{\mu}\right)$ respectively, depending continously on $\mu$, as $C^{1}$ submanifolds with boundary of $R^{n}$. The point $p_{\mu}$ does not necessarily belong to $A_{\mu}^{u}$ or $A_{\mu}^{s}$.

Definition 1.9. - The arc $\left\{G_{\mu}\right\}, \mu \in[a, b]$ in $\mathcal{H}_{D}$ exhibits a homoclinic bifurcation with unavoidable tangency if there exist $p_{\mu}, A_{\mu}^{u}, A_{\mu}^{s}$ as above, such that:

(i) $\partial A_{\mu}^{u} \cap A_{\mu}^{s}=\partial A_{\mu}^{s} \cap A_{\mu}^{u}=\emptyset$, for all $\mu \in[a, b]$

(ii) $A_{a}^{u} \cap A_{a}^{s}=\emptyset$

(iii) $A_{b}^{u} \cap A_{b}^{s}$ contains at least one point of transversal intersection.

The name unavoidable tangency of the definition above is due to the following:

Proposition 1.10. - If $\left\{G_{\mu}\right\}, \mu \in[a, b]$, is an arc as in the definition 1.9 , then there exists $\mu_{0} \in[a, b]$ such that $G_{\mu_{0}}$ has a periodic point with a homoclinic tangency.

Proof. - As the interval $[a, b]$ is connected, there exists $\mu_{0} \in(a, b)$ such that $A_{\mu}^{s}$ and $A_{\mu}^{u}$ have a non transversal intersection. It must be a tangency because the dimension of $A_{\mu}^{u}$ is one.

We will take the definition of band-merging maps from [5], and relate it with the homoclinic bifurcations.

Let $\hat{M}$ be a manifold of one-dimensional maps defined at the beginning of the subsection 1.1.

DEFINITION 1.11. - A map $\psi \in \hat{M}$ is band-merging if: $0<\psi \circ \psi(1)=$ $-\psi(1)<1$.

As $\psi(x)=g\left(x^{2}\right)$, we have the following equivalent definition: $0<g\left((g(1))^{2}\right)=-g(1)<1$. As $g(0)=1$ and $g(1)<0, g^{\prime}<0$ and so $x \psi(x)<0$ for all $x \neq 0$. 
PROPOSITION 1.12. - If $\psi$ is band-merging and the Schwarzian derivative $S \psi$ is negative then:

a) $-\psi(1)$ is a hyperbolic repellor, whose repelling basin includes $[\psi(1),-\psi(1)]$,

b) any $\tilde{\psi} \in \hat{M}$, near enough $\psi$, has a repelling fixed point whose basin includes $[\tilde{\psi}(1),-\tilde{\psi}(1)]$.

Proof. - Let us see part a).

The map $\psi \circ \psi$ is increasing in $\left(0, x_{-1}\right)$, where $x_{-1}>0$ and $\psi\left(x_{-1}\right)=0$. Its graph, at $x=0$ is below the diagonal, at $x_{-1}$ is above the diagonal, and at $x_{0}=-\psi(1) \in\left(0, x_{-1}\right)$ intersects the diagonal. By contradiction, suppose that $(\psi \circ \psi)^{\prime}\left(x_{0}\right) \leq 1$. Then, there exists $x_{1}$ where $(\psi \circ \psi)^{\prime \prime}$ vanishes and $(\psi \circ \psi)^{\prime \prime \prime}$ is non negative. This implies that $S(\psi \circ \psi)\left(x_{1}\right) \geq 0$, contradicting our hypothesis, because $S \psi<0$ implies $S(\psi \circ \psi)<0$. The same contradiction is obtained if $\psi \circ \psi$ is supposed to have other fixed point $\bar{x}_{0} \in\left[0, x_{0}\right)$. Therefore $x_{0}$ is a repellor and $\left[0, x_{0}\right]$ is in its basin. By symmetry, also $\left[-x_{0}, 0\right]$ is.

To show part $b$ ), consider any $\tilde{\psi}$ near enough $\psi$, so that it also has a hyperbollic repellor, and $S \tilde{\psi}<0$. The proof also works for $\tilde{\psi}$ instead of $\psi$.

Due to the above proposition, the band merging maps with negative Schwartzian derivative satisfy the condition that the critical point lands after three iterations on the unstable periodic point.

We recall that the family $\left\{\varphi_{t}\right\}$ is the unstable manifold in $\hat{M}$ of the hyperbolic fixed point $\varphi$ of the renormalization $\hat{\mathcal{F}}$. It is parametrized such that $\varphi_{0}=\varphi, \hat{\mathcal{F}}\left(\varphi_{t}\right)=\varphi_{\delta t}$ and $\varphi_{-1} \in \hat{\Sigma}_{1}$.

THEOREM 1.13 (Eckmann and Wittwer). - There exists $t_{0}>0$ such that $\varphi_{t_{0}} \in \hat{\mathcal{W}}^{u}(\varphi)$ is band-merging, and for all $t$ near $t_{0}$ :

$$
\frac{\partial}{\partial t}\left(\varphi_{t}\left(\varphi_{t}(1)\right)+\varphi_{t}(1)\right)<0
$$

Proof. - See [5].

This last theorem asserts that $\hat{\mathcal{W}}^{u}(\varphi)$ at $\varphi_{t_{0}}$ intersects transversally in $\hat{M}$ the codimension one (in $\hat{M}$ ) manifold of band-merging maps.

Now let us consider the family of maps $\left\{\Phi_{t}\right\}$ in $\mathcal{H}_{D}$, that is $\mathcal{W}^{u}(\Phi)$, the unstable manifold of $\Phi$ by the renormalization $T$. Due to part $c$ ) of the corollary 1.7 it is obtained from $\left\{\varphi_{t}\right\}$.

The following lemma is a consequence of the theorem 1.13. 
LEMMA 1.14. - Given $\varepsilon>0$, for all $\gamma>0$ sufficiently small the arc $\left\{\Phi_{t}\right\}, t \in\left[t_{0}-\gamma, t_{0}+\gamma\right]$ exhibits a homoclinic bifurcation with unavoidable tangency, and the first coordinate projection of the compact part $A_{t}^{s}$ (cf. definition 1.9) is contained in $(-\varepsilon, \varepsilon)$.

Proof. - First, we assert that $\Phi_{t_{0}}=\left(f_{t_{0}} \circ \theta, 0\right)$ has a hyperbolic fixed point $p_{t_{0}}=\left(-f_{t_{0}}(1), \mathbf{0}\right)$ of stable codimension one. In fact, it is fixed because $f_{t_{0}} \circ \theta_{0}$ is band merging. Let us see that it is hyperbolic, computing $D \Phi_{t_{0}}\left(p_{t_{0}}\right)$ :

$$
D \Phi_{t_{0}}=\left[\begin{array}{cc}
2 x f_{t_{0}}^{\prime} \circ \theta & -\left(f_{t_{0}}^{\prime} \circ \theta\right) \cdot \alpha \\
0 & \mathbf{0}
\end{array}\right]
$$

with $2 x f_{t_{0}}^{\prime}\left(x^{2}\right)=\left(f_{t_{0}} \circ \theta_{0}\right)^{\prime}(x)$. But $f_{t_{0}} \circ \theta_{0}$ belongs to the unstable manifold $\hat{\mathcal{W}}^{u}(\varphi)$ in $\hat{M}$, and all maps in $\hat{\mathcal{W}}^{u}(\varphi)$ have negative Schwarzian derivative because all the maps in a neighborhood of $\varphi$ have, and also their renormalizations. Therefore, proposition 1.12 states that $-f_{t_{0}}(1)$ is a repellor. Thus:

$$
\left|2 x_{0} f_{t_{0}}^{\prime}\left(x_{0}^{2}\right)\right|>1 \text { for } x_{0}=-f_{t_{0}}(1)
$$

Thus, our assertion is proved.

Let us choose $\gamma>0$ small enough so that, for all $l \in\left[\iota_{0}-\gamma, \iota_{0}+\gamma\right]$ there exists $p_{t}=\left(x_{t}, \mathbf{0}\right)$, continuation of $p_{t_{0}}$, hyperbolic fixed point of $\Phi_{t}=\left(f_{t} \circ \theta, \mathbf{0}\right) \in \mathcal{W}^{u}(\Phi)$. Here $x_{t}$ is the hyperbolic repellor of the unimodal map $\varphi_{t}=f_{t} \circ \theta_{0}$, whose repelling basin includes $\left[f_{t}(1),-f_{t}(1)\right]$, as proved in the proposition 1.12 .

We define:

$$
A_{t}^{u}=\left\{(x, X): X=\mathbf{0},|x| \leq-f_{t}(1)\right\} \subset W^{u}\left(p_{t}\right)
$$

The theorem 1.13 allows us to choose $\gamma$ such that $\varphi_{t}\left(\varphi_{t}(1)\right)+\varphi_{t}(1)$ is positive for $t \in\left[t_{0}-\gamma, t_{0}\right)$ and negative for $t \in\left(t_{0}, t_{0}+\gamma\right]$.

We assert that given $\delta>0$ there exists $\gamma$ sufficiently small and $y_{t} \in \varphi_{t}^{-2}\left(x_{t}\right)$, for all $t \in\left[t_{0}-\gamma, t_{0}+\gamma\right]$, such that:

$$
\begin{aligned}
& 1<y_{t}<1+\delta \text { if } t \in\left[t_{0}-\gamma, t_{0}\right) \\
& y_{t_{0}}=1 \\
& 1-\delta<y_{t}<1 \text { if } t \in\left(t_{0}, t_{0}+\gamma\right]
\end{aligned}
$$

In fact, if $t \in\left[t_{0}-\gamma, t_{0}\right)$ we have $\varphi_{t}\left(\varphi_{t}(1)\right)+\varphi_{t}(1)>0$, i.e. the graph of $\varphi_{t}$ at $-\varphi_{t}(1)$ is above the diagonal. As $\varphi_{t}$ is decreasing in $(0,1]$, the fixed point $x_{t}$ is at right of $-\varphi_{t}(1)$. Therefore, given $\delta_{1}>0$ :

$$
\varphi_{t}(1)>-x_{t}>\varphi_{t}(1)-\delta_{1}
$$

for all $t \in\left[t_{0}-\gamma, t_{0}\right)$, near enough $t_{0}$. 
The map $\varphi_{t}$ is decreasing at right of 0 and defined in a neighborhood $D$ of $[-1,1]$. We conclude that, given $\delta>0$, there exists $\gamma$ and $y_{t} \in \varphi_{t}^{-1}\left(-x_{t}\right)$ for all $t \in\left[t_{0}-\gamma, t_{0}\right)$, such that $1<y_{t}<1+\delta$. As $-x_{t} \in \varphi_{t}^{-1}\left(x_{t}\right)$, we have $y_{t} \in \varphi_{t}^{-2}\left(x_{t}\right)$. The same argument, with the opposite inequalities, is valid for $t \in\left(t_{0}, t_{0}+\gamma\right]$. This completes the proof of our assertion.

We have $f_{t}(0)=1$ and $f_{t}^{\prime}(0)<0$. If $\gamma$ is small, for any $t \in\left[t_{0}-\gamma, t_{0}+\gamma\right]$, the map $f_{t}$ is invertible and decreasing in a fixed neighborhood of 0 . Let us denote $\varepsilon_{t}=f_{t}^{-1}\left(y_{t}\right)$. Our previous assertion can be reformulated as follows:

Given $\varepsilon>0$, for all $\gamma$ sufficiently small:

$$
\begin{aligned}
& -\varepsilon^{2}<\varepsilon_{t}<0 \text { if } t \in\left[t_{0}-\gamma, t_{0}\right) \\
& \varepsilon_{t_{0}}=0 \\
& 0<\varepsilon_{t}<\varepsilon^{2} \text { if } t \in\left(t_{0}, t_{0}+\gamma\right]
\end{aligned}
$$

With no loss of generality, let us suppose that $\alpha_{n-1} \neq 0$. (Recall that $\left.\alpha=\left(\alpha_{1}, \alpha_{2}, \ldots, \alpha_{n-1}\right) \neq \mathbf{0}\right)$.

Let us denote $X=\left(X_{1}, X_{2}, \ldots, X_{n-1}\right)$. Now we can define, for given $\varepsilon>0$, :

$$
A_{t}^{s}=\left\{(x, X): x^{2}-\alpha X=\varepsilon_{t},|x| \leq \varepsilon,\left\|\left(X_{1}, \ldots, X_{n-2}\right)\right\| \leq \varepsilon\right\} .
$$

It is easy to check that $A_{t}^{s} \subset \Phi_{t}^{-3}\left(p_{t}\right) \subset W^{s}\left(p_{t}\right)$.

Let us see how $A_{t}^{s}$ looks: For $t=t_{0}, \varepsilon_{t_{0}}=0$ and $A_{t_{0}}^{s} \subset\left\{x^{2}-\alpha X=0\right\}$. It is a quadratic codimension one manifold of $\mathbf{R}^{n}$, passing through $(0, \mathbf{0})$ and tangent at $(0,0)$ to $A_{t_{0}}^{u}$. For $t \in\left[t_{0}-\gamma, t_{0}\right), \varepsilon_{t}<0$, and $A_{t}^{s}$ does not intersect $\{X=\mathbf{0}\} \supset A_{t}^{u}$. For $t \in\left(t_{0}, t_{0}+\gamma\right], \varepsilon_{t} \in\left(0, \varepsilon^{2}\right)$. So $A_{t}^{s}$ intersects $\{X=\mathbf{0}\}$ at two points $q=\left(-\sqrt{\varepsilon_{t}}, \mathbf{0}\right)$ and $r=\left(\sqrt{\varepsilon_{t}}, \boldsymbol{0}\right)$, both in the $\varepsilon$-neighborhood of $(0,0)$. Then both $q$ and $r$ are in $A_{t}^{u}$.

Moreover $T_{q} A_{t}^{s}$ and $T_{r} A_{t}^{s}$ are transversal to the subspace $\{X=\mathbf{0}\}=$ $T_{q} A_{t}^{u}=T_{r} A_{t}^{u}$.

Finally, if $\varepsilon$ is small enough, we get $\partial A_{t}^{s} \cap A_{t}^{u}=\partial A_{t}^{u} \cap A_{t}^{s}=\emptyset$, for all $t \in\left[t_{0}-\gamma, t_{0}+\gamma\right]$.

Now we are ready to perturb the family $\left\{\Phi_{t}\right\}, t \in\left[t_{0}-\gamma, t_{0}+\gamma\right]$, contained in $\mathcal{W}^{u}(\Phi)$, and prove that the homoclinic bifurcation persists for nearby families.

LEMMA 1.15 - There exists an interval $[a, b]$ with $b>a>0$ and neighborhoods $N, N_{1}$ and $N_{2}$ in $\mathcal{H}_{D}$, of $\left\{\Phi_{t}: t \in[a, b]\right\}, \Phi_{a}$ and $\Phi_{b}$ respectively, such that any continuous arc $\left\{G_{\mu}\right\}$, in $N$, with extremities in $N_{1}$ and $N_{2}$, exhibits a homoclinic bifurcation with unavoidable tangency. 
Proof. - Let us take $\Phi_{t_{0}}=\left(f_{t_{0}} \circ \theta, 0\right)$ with $t_{0}$ as in the theorem 1.13. We have that $p_{t_{0}}=\left(-f_{t_{0}}(1), \mathbf{0}\right)$ is a fixed point, of saddle type. Its local stable manifold is contained in $\left\{(x, X): x^{2}-\alpha X-\left(f_{t_{0}}(1)\right)^{2}=0\right\}$. Any $G$ in a small neighborhood of $\Phi_{t_{0}}$ in $\mathcal{H}_{D}$, has an hyperbolic fixed point $p(G)$, whose local stable manifold is of codimension one, given in a neighborhood of $p(G)$ by the equation

$$
\{(x, X): U(x, X, G)=0\}
$$

where $U(\cdot, \cdot, G)$ is a smooth real function of $(x, X)$, depending continously on $G$ ([8], [14]). We have $U\left(x, X, \Phi_{t_{0}}\right)=x^{2}-\alpha X-\left(f_{t_{0}}(1)\right)^{2}$.

Let us define, for any $(x, X, G)$ in a certain small neighborhood of $\left(0, \mathbf{0}, \Phi_{t_{0}}\right)$ in $\mathbf{R}^{n} \times \mathcal{H}_{D}$, the real function:

$$
F(x, X, G)=U\left(G^{3}(x, X), G\right)
$$

The point $(0, \mathbf{0})$ verifies $\Phi_{t_{0}}^{3}(0, \mathbf{0})=p_{t_{0}}$, and so

$$
F\left(0, \mathbf{0}, \Phi_{t_{0}}\right)=0
$$

As in the proof of the previous lemma, let us suppose $\alpha_{n-1} \neq 0$, and compute the partial derivative:

$$
\frac{\partial F}{\partial X_{n-1}}\left(0, \mathbf{0}, \Phi_{t_{0}}\right)=2 \alpha_{n-1} f_{t_{0}}(1)\left(\varphi_{t_{0}}^{2}\right)^{\prime}(1) f_{t_{0}}^{\prime}(0) \neq 0
$$

Now, by the implicit function theorem, there exists $N_{0}$, neighborhood of $\Phi_{t_{0}}$ in $\mathcal{H}_{D}$, and $\varepsilon>0$ such that for all $G \in N_{0}$, for all $x \in B_{\varepsilon}(0)$ and for all $\left(X_{1}, \ldots, X_{n-2}\right)$ in $\mathbf{R}^{n-2}$ with norm less than $\varepsilon$, is defined the coordinate $X_{n-1}=u\left(x, X_{1}, \ldots, X_{n-2}, G\right)$ verifying:

$$
(x, X) \in G^{-3}\left(W_{l o c}^{s}(p(G))\right) \subset W^{s}(p(G))
$$

with $X=\left(X_{1}, \ldots, X_{n-1}\right)$.

Let us take

$$
\begin{aligned}
A^{s}(G)= & \left\{(x, X): X_{n-1}=u\left(x, X_{1}, \ldots, X_{n-2}, G\right)\right. \\
& \left.|x| \leq \varepsilon ;\left\|\left(X_{1}, \ldots, X_{n-2}\right)\right\| \leq \varepsilon\right\}
\end{aligned}
$$

We have that $A^{s}(G)$ is a $C^{1}$ submanifold with boundary of $\mathbf{R}^{n}$, that depends continously on $G \in N_{0}$. It is a compact part of $W^{s}(p(G))$. For the neighborhood $N_{0}$ and $\varepsilon>0$ as above, let us take $\gamma$ as in the previous 
lemma, and also such that $\Phi_{t} \in N_{0} \forall t \in[a, b]=\left[t_{0}-\gamma, t_{0}+\gamma\right]$. We take $A_{t}^{s}=A^{s}\left(\Phi_{t}\right)$ and $A_{t}^{u}$ as in the proof of the previous lemma. $A_{t}^{u}$ is contained, for some fixed $n_{0}$ independent of $t$, in $\Phi_{t}^{n_{0}}\left(W_{l o r}^{u}\left(p\left(\Phi_{t}\right)\right)\right)$. Let us take for any $t \in[a, b]$, a small neighborhood $N_{t}$ of $\Phi_{t}$ in $N_{0}$, such that $G^{n_{0}}\left(W_{l o c}^{u}(p(G))\right)$ is $C^{1}$ near to $\Phi_{t}^{n_{0}}\left(W_{l o c}^{u}\left(p\left(\Phi_{t}\right)\right)\right)$, for any $G \in N_{t}$. Consequently, compact parts $A^{u}(G)$ and $A^{s}(G)$ can be chosen, proximate to $A_{t}^{u}$ and $A_{t}^{s}$ respectively as $C^{1}$ submanifolds with boundary, for any $G \in N_{t}$. The three conditions in the definition 1.9 are persistent under small $C^{1}$ perturbations of $A_{t}^{u}$ and $A_{t}^{s}$. Therefore, the lemma is proved taking

$$
N=\bigcup_{t \in[a, b]} N_{t} ; \quad N_{1}=N_{a} ; \quad N_{2}=N_{b}
$$

Now, we are ready to complete the proof of the theorem 1.

Proof of part $b$ ) of theorem 1. - Let $\mathcal{W}=\mathcal{W}^{s}(\Phi)$. The lemma above states the existence of the arc $\left\{\Phi_{t}: t \in[a, b]\right\} \subset \mathcal{W}^{u}(\Phi)$ and the neighborhoods $N, N_{1}, N_{2}$. Given a curve $\left\{G_{\mu}\right\}$, transversal at $\mu=0$ to $\mathcal{W}$, its images by the renormalization $T^{m}$ accumulate, when $m \rightarrow \infty$, at the unstable manifold of $\Phi$, due to the inclination lemma [14]. In particular they approach the arc $\left\{\Phi_{t}: t \in[a, b]\right\} \subset \mathcal{W}^{u}(\Phi)$. Consequently, there exists $\left[a_{m}, b_{m}\right]$, for all $m$ sufficiently large, such that $T^{m} G_{a_{m}} \in N_{1}, \quad T^{m} G_{b_{m}} \in N_{2}, \quad T^{m} G_{\mu} \in N$ for all $\mu \in\left[a_{m}, b_{m}\right]$.

Besides $\left[a_{m}, b_{m}\right] \rightarrow 0$, because the argument above works for any subarc of $\left\{G_{\mu}\right\}$ as near as wanted from $G_{0}$.

The lemma 1.15 states that $\left\{T^{m} G_{\mu}\right\}, \mu \in\left[a_{m}, b_{m}\right]$ exhibits a homoclinic bifurcation with unavoidable tangency, and so there exists $\bar{\mu}_{m} \in\left[a_{m}, b_{m}\right] \rightarrow 0$ with $G_{\bar{\mu}_{m}}$ exhibiting a homoclinic tangency.

The part $a$ ) of the theorem 1 states the existence of $\mu_{m} \rightarrow 0$ where $G_{\mu_{m}}$ is period doubling bifurcating. As the intersection of $\Sigma_{1}$ with $\mathcal{W}^{u}(\Phi)$ is produced at $\Phi_{-1}$ and the homoclinic tangencies were found in a neighborhood $N$ of $\left\{\Phi_{t}\right\}_{t \in[a, b]}$ with $b>a>0$, it follows that $\bar{\mu}_{m}$ is at the other side of 0 than $\mu_{m}$.

\section{THE $C^{r}$ THEORY}

Now we will work in a $C^{r}$ neighborhood $\mathcal{U}$ of $\Phi_{0}(r \geq 3)$. We define, for $G \in \mathcal{U}$

$$
\mathcal{F}^{i}(G)=\Lambda_{i, G}^{-1} \circ G^{2^{i}} \circ \Lambda_{i, G}
$$


where $\Lambda_{i, G} \in \mathcal{L}\left(\mathbf{R}^{n}, \mathbf{R}^{n}\right)$ is defined as $\Lambda_{i, G}\left(z_{0}, Z\right)=\left(\lambda_{i, G} z_{0}, \lambda_{i, G}^{2} Z\right)$ with $\lambda_{i, G}=\frac{\pi_{1} \circ G^{2^{i}}(0,0)}{\pi_{1} \circ G(0,0)}$.

To obtain good spectral properties we need to redefine the renormalization for $G \in \mathcal{U}$, considering a sequence $\left\{T_{i}\right\}_{i \in Z^{+}}$, where

$$
T_{i}(G)=\left(I-\sigma\left[\mathcal{F}^{i}(G)-\Phi_{0}\right]\right)^{-1} \circ \mathcal{F}^{i}(G) \circ\left(I-\sigma\left[\mathcal{F}^{i}(G)-\Phi_{0}\right]\right)
$$

We need first to extend the linear operator $\sigma$, using the spectral projection, which is defined, up to the moment, only for the space $\mathcal{H}_{D}$ of real-analytic maps.

The main problem that arises in the $C^{r}$ topology is that the renormalization is not Fréchet differentiable. But it is in the space $\mathcal{H}_{D}$ and its derivative, computed at an analytic map, can be extended to a bounded linear operator on the space of $C^{r}$ maps. We will work with these operators called formal derivatives. On the other hand the transformation $T_{i}$ is Fréchet differentiable when considered from the space of $C^{r}$ maps to the space of $C^{r-1}$ maps. We will work with its derivatives in this sense too, computed at maps of class $C^{r}$. Finally these derivatives can be also extended to bounded linear operators on the space of $C^{r-1}$ or $C^{r-2}$ maps as formal derivatives. On each case the sense in which a functional derivative (formal or Fréchet) is considered will be explicited or otherwise clear from the context.

The unstable manifold found in the space of real-analytic maps verifies $T_{i}\left(\left\{\Phi_{t}\right\}\right)=\left\{\Phi_{t}\right\}$, now immersed in the space $C^{r}$. The purpose of this section is to define $T_{i}$ for $C^{r}$ maps and then to prove in the $C^{r}$-topology that the distance of a renormalizable $C^{r}$ map $G$ in $\mathcal{U}$ to the manifold $\left\{\Phi_{t}\right\}_{t}$ decreases. More precisely, we prove the following

PRoposition 2.1. - For $r$ large enough there exist $\zeta>0$ and an integer $N$ as large as wanted such that for all real $t$ with $|t|<\zeta$ and all $C^{r}$ map $u$ with $\|u\|_{r}<\zeta$

$$
\left\|T_{N}\left(\Phi_{t}+u\right)-\Phi_{s}\right\|_{r} \leq \frac{1}{2}\|u\|_{r}
$$

where $s=\delta^{N}(t+a(u))$, and a is a bounded linear operator from $C^{r}$ to $\mathbf{R}$.

This proposition is an extension to $n$-dimensional maps of the lemma 8 of the paper [4].

We begin giving some definitions.

Let us consider a compact parallelepiped $D$ whose sides are parallel to the coordinates axes, which is a neighborhood of $[-1,1] \times\{\mathbf{0}\}$ in $\mathbf{R}^{n}$, and 
the Banach space $C^{r}$ of maps from $D$ to $\mathbf{R}^{n}$, of class $C^{r}$ with the norm $\|\cdot\|_{r}$ For a given number $\eta>0$, let us define the localized seminorm

$$
\|G\|_{r, \eta}=\sup _{\|x-y\|<\eta}\left\|D^{r} G(x)-D^{r} G(y)\right\|
$$

Let $\mathcal{S}$ be the bounded linear operator on $C^{r}$ defined as

$$
\mathcal{S} G(x)=\sum_{i=1}^{k} a_{i}(x) \cdot G\left(b_{i}(x)\right)
$$

where $b_{i} \in C^{r+1}, b_{i}(D) \subset D$; and for each $x \in D, a_{i}(x)$ is a matrix $n \times n$, depending $C^{r+1}$ of $x$.

Associated to $\mathcal{S}$, let $\mathcal{S}_{r}$ be the operator defined on the continuous functions from $D$ to $\mathcal{L}_{r}\left(\mathbf{R}^{n}, \mathbf{R}^{n}\right)$ (the set of $r$-linear applications from $\mathbf{R}^{n}$ on $\mathbf{R}^{n}$ ) defined as follows:

$$
\left(\mathcal{S}_{r} H\right)(x)=\sum_{i=1}^{k}\left\|a_{i}(x)\right\|\left\|D b_{i}(x)\right\|^{r} H\left(b_{i}(x)\right)
$$

where $\left\|a_{i}(x)\right\|$ is the norm of the $n \times n$ matrix $a_{i}(x)$ as linear operator in $\mathbf{R}^{n}$ (it is a function of $x$ ), and similarly we define $\left\|D b_{i}(x)\right\|$.

Lemma 2.2. - Let $\rho>0$. If the spectral radius of $\mathcal{S}_{r}$ is less than $\rho$, then for any $\varepsilon>0$ there exists a positive integer $N_{0}$ such that for all $N \geq N_{0}$ and for some $\eta_{0}=\eta_{0}(\varepsilon, N)$ we have $\left\|\mathcal{S}^{N} G\right\|_{r, \eta} \leq \varepsilon \rho^{N}\|G\|_{r}$ for all $G \in C^{r}$, and $\eta \leq \eta_{0}$.

Proof. - First observe that $\mathcal{S}^{N} G(x)$ can be written as

$$
\sum_{i=1}^{k^{N}} u_{i}(x) G\left(v_{i}(x)\right)
$$

with $u_{i}(x) \in \mathcal{L}\left(\mathbf{R}^{n}, \mathbf{R}^{n}\right)$, depending $C^{r+1}$ on $x ; v_{i} \in C^{r+1}$ such that $v_{i}(D) \subset D$. Also,

$$
\left(\left(\mathcal{S}^{N}\right)_{r} H\right)(x)=\sum_{i=1}^{k^{N}}\left\|u_{i}(x)\right\|\left\|D v_{i}(x)\right\|^{r} H\left(v_{i}(x)\right)
$$

We choose $\rho^{\prime}<\rho, \rho^{\prime}$ greater than the spectral radius of $\mathcal{S}_{r}$. Thus, $\left\|\left(\mathcal{S}_{r}\right)^{N}\right\|<\rho^{\prime N}<\frac{\varepsilon \rho^{N}}{12}$ for $N$ large enough, being $\left\|\left(\mathcal{S}_{r}\right)^{N}\right\|$ the norm Vol. $15, \mathrm{n}^{\circ} 3-1998$ 
of $\left(\mathcal{S}_{r}\right)^{N}$ as linear operator. As $\left\|\left(\mathcal{S}^{N}\right)_{r}\right\| \leq\left\|\left(\mathcal{S}_{r}\right)^{N}\right\|$, taking $H=$ id we have that

$$
\sum_{i=1}^{k^{N}}\left\|u_{i}(x)\right\|\left\|D v_{i}(x)\right\|^{r}<\frac{\varepsilon \rho^{N}}{12}
$$

We agree into that, in what follows, $K$ is a constant whose value may vary in the different formulas.

We have to bound the localized seminorm of the $r$-th derivative of $\mathcal{S}^{N} G$. $D^{r}\left(\mathcal{S}^{N} G\right)(x)$ is a $r$-linear transformation, computed as follows:

$$
D^{r}\left(\mathcal{S}^{N} G\right)(x)=\sum_{i=1}^{k^{N}} D^{r}\left[u_{i}(x) \cdot G\left(v_{i}(x)\right)\right]=\sum_{i=1}^{k^{N}} u_{i}(x) \cdot\left(v_{i}^{*} D^{r} G\right)(x)+R(x)
$$

where $\left(v_{i}^{*} D^{r} G\right)(x) \cdot\left(e_{1}, \ldots, e_{r}\right)=D^{r} G\left(v_{i}(x)\right) \cdot\left(D v_{i}(x) e_{1}, \ldots, D v_{i}(x) e_{r}\right)$; and $R(x)$ involves derivatives of $G$ of order smaller than $r$ and derivatives up to $r$ of $u_{i}$ and $v_{i}$ that are $C^{1}$.

$$
\begin{gathered}
\left\|\mathcal{S}^{N} G\right\|_{r, \eta}=\sup _{\|x-y\|<\eta}\left\|D^{r} \mathcal{S}^{N} G(x)-D^{r} \mathcal{S}^{N} G(y)\right\| \leq \\
\leq \sup _{\|x-y\|<\eta} \sum_{i=1}^{k^{N}}\left\|u_{i}(x)\left(v_{i}^{*} D^{r} G\right)(x)-u_{i}(y)\left(v_{i}^{*} D^{r} G\right)(y)\right\|+\|R(x)-R(y)\|
\end{gathered}
$$

The last term is bounded as follows:

$$
\|R(x)-R(y)\| \leq K\|G\|_{r}\|x-y\| \leq K \eta\|G\|_{r} \leq \frac{\varepsilon}{2} \rho^{N}\|G\|_{r}
$$

if $\eta$ is small enough.

The first term can be bounded by the sum of the following:

$$
\begin{gathered}
A=\sum_{i=1}^{k^{N}} \| u_{i}(x)\left(D^{r} G\left(v_{i}(x)\right)-D^{r} G\left(v_{i}(y)\right) \cdot\left(D v_{i}(x) \cdot, \ldots, D v_{i}(x) \cdot\right) \|\right. \\
B_{1}=\sum_{i=1}^{k^{N}}\left\|u_{i}(x) D^{r} G\left(v_{i}(y)\right) \cdot\left(\left(D v_{i}(x)-D v_{i}(y)\right) \cdot, \ldots, D v_{i}(x) \cdot\right)\right\| \\
\vdots \\
B_{r}=\sum_{i=1}^{k^{N}}\left\|u_{i}(x) D^{r} G\left(v_{i}(y)\right) \cdot\left(D v_{i}(y) \cdot, \ldots,\left(D v_{i}(x)-D v_{i}(y)\right) \cdot\right)\right\| \\
C=\sum_{i=1}^{k^{N}}\left\|\left(u_{i}(x)-u_{i}(y)\right) D^{r} G\left(v_{i}(y)\right) \cdot\left(D v_{i}(y) \cdot, \ldots, D v_{i}(y) \cdot\right)\right\|
\end{gathered}
$$


Now, using that $u_{i}$ and $v_{i}$ are of class $C^{r+1}$ and taking $\eta$ small enough:

$$
\begin{gathered}
C \leq \sum_{i=1}^{k^{N}} K\|x-y\|\left\|D^{r} G\right\|_{0}\left\|D v_{i}\right\|_{0}^{r} \leq K \eta\|G\|_{r} \leq \frac{\varepsilon}{6} \rho^{N}\|G\|_{r} \\
B_{j} \leq \sum_{i=1}^{k^{N}}\left\|u_{i}\right\|_{0}\left\|D^{r} G\right\|_{0}\left\|D v_{i}\right\|_{0}^{r-1} K\|x-y\| \leq K \eta\|G\|_{r} \leq \frac{\varepsilon}{6 r} \rho^{N}\|G\|_{r} \\
A \leq \sum_{i=1}^{k^{N}}\left\|u_{i}(x)\right\|\left\|D v_{i}(x)\right\|^{r}\left\|D^{r} G\left(v_{i}(x)\right)-D^{r} G\left(v_{i}(y)\right)\right\| \leq \\
\leq \sum_{i=1}^{k^{N}}\left\|u_{i}(x)\right\|\left\|D v_{i}(x)\right\|^{r} 2\|G\|_{r} \leq \frac{\varepsilon}{6} \rho^{N}\|G\|_{r}
\end{gathered}
$$

In the last inequality we have used (3). Thus, $A+\sum_{j=1}^{r} B_{j}+C \leq \frac{\varepsilon}{2} \rho^{N}\|G\|_{r}$ and $\left\|\mathcal{S}^{N} G\right\|_{r, \eta} \leq \varepsilon \rho^{N}\|G\|_{r}$ as wanted.

The last lemma allows us to bound $\left\|\mathcal{S}^{N} G\right\|_{r, \eta}$ knowing a spectral bound of the associated operator $\mathcal{S}_{r}$. The following lemma connects the $r$-norm with the localized seminorm:

LEMMA 2.3. - Let $\eta>0$ be a sufficiently small real number. There exists $c=c(n, r)$ such that for all $G \in C^{r}$,

$$
\|G\|_{r} \leq 2 c \max \left\{\|G\|_{r, \eta}, \frac{2}{\eta}\|G\|_{r-1}\right\}
$$

Proof. - Let us make explicit the computations for $n=2$ but observing that similar considerations can be made for $n>2$. Let us denote $G_{x^{i}, y^{j}}=\frac{\partial^{i+j} G}{\partial x^{i} \partial y^{j}}$, for $i+j=r, \pi_{1}(x, y)=x ; \pi_{2}(x, y)=y$. We have for $i \geq 1, k \in\{1,2\}$ :

$$
\int_{x_{0}}^{x} \pi_{k} G_{x^{i}, y^{j}}(t, y) d t=\pi_{k} G_{x^{i-1}, y^{i}}(x, y)-\pi_{k} G_{x^{i-1}, y^{j}}\left(x_{0}, y\right)
$$

We have a similar equality for $j \geq 1$, integrating respect to the second variable. The right member of the equality is bounded in absolute value by $2\|G\|_{r-1}$.

Let $c \geq 1$ be a constant such that for every $G$ of class $C^{r}$

$$
\left\|D^{r} G(x, y)\right\| \leq c \max _{i+j=r ; k \in\{1,2\}}\left|\pi_{k} G_{x^{i}, y^{j}}\right|
$$


It is enough to prove that $\|G\|_{r} \leq 2 c$ for all $G \in C^{r}$ such that

$$
\max \left\{\|G\|_{r, \eta},(2 / \eta)\|G\|_{r-1}\right\}=1
$$

By contradiction suppose that we have a point $z_{0}=\left(x_{0}, y_{0}\right), k \in\{1,2\}$ and $i$ and $j$ with $i+j=r$ such that $\left|\pi_{k} G_{x^{i}, y^{j}}\left(z_{0}\right)\right|>2$. Thus, as $\left\|D^{r} G(z)-D^{r} G\left(z_{0}\right)\right\| \leq 1$ for $z \in D$ in a ball centered in $z_{0}$ of radius $\eta$, we have

$$
\left|\pi_{k} G_{x^{i}, y^{j}}(z)\right| \geq\left|\pi_{k} G_{x^{i}, y^{j}}\left(z_{0}\right)\right|-\left\|D^{r} G\left(z_{0}\right)-D^{r} G(z)\right\|>1
$$

Integrating up to the boundary of the ball of radius $\eta$, the absolute value of the left term of the equality (4) is greater than $\eta$. The absolute value of the right term is smaller than $\eta$ because $\|G\|_{r-1} \leq \eta / 2$. This contradiction proves the lemma.

We recall the equation (1) from the analytic theory in the subsection 1.1. For $u=\left(u_{0}, U\right) \in \mathcal{H}_{D}$ we have

$$
\begin{aligned}
& d \mathcal{F}\left(\Phi_{0}\right)(u)=\Lambda^{-1}\left[u \circ \Phi_{0} \circ \Lambda+\left(D \Phi_{0} \circ \Phi_{0} \circ \Lambda\right) \circ(u \circ \Lambda)\right] \\
& \quad+\left(\frac{u_{0}(1, \mathbf{0})}{\lambda}+u_{0}(0, \mathbf{0})\left(\frac{1}{\lambda^{2}}-1\right)-\frac{\alpha \cdot U(0, \mathbf{0})}{2 \lambda^{2}}\right) \Psi_{\sigma_{1}}
\end{aligned}
$$

for $\Psi_{\sigma_{1}}=-\sigma_{1} \circ \Phi_{0}+D \Phi_{0} \cdot \sigma_{1}$, and $\sigma_{1}(x, Y)=(x, 2 Y)$. Now, if $u \in C^{r}$, the last operator can be extended to a bounded operator $F$, defining $F u$ as the right term of (5). The associated operator $F_{r}$ is

$$
F_{r} H=|\lambda|^{-2}\left\|\left(D \Phi_{0} \circ \Lambda\right) \Lambda\right\|^{r} H \circ \Phi_{0} \circ \Lambda+|\lambda|^{r}\left\|\Lambda^{-1} D \Phi_{0} \circ \Phi_{0} \circ \Lambda\right\| H \circ \Lambda
$$

LeMma 2.4. - The spectral radius of $F_{r}$ converges to 0 when $r$ goes to infinite. As a consequence, there exists $r_{0}$ large enough such that the spectral radius is smaller than 1.

Proof. - The spectral radius is bounded by the norm of the operator $F_{r}$.

$$
\begin{gathered}
\left\|F_{r}\right\| \leq \sup _{(x, Y) \in D}\left\{|\lambda|^{r}\left(|\lambda|^{-2}\left\|D \Phi_{0} \circ \Lambda(x, Y)\right\|^{r}+\left\|\Lambda^{-1} D \Phi_{0} \circ \Phi_{0} \circ \Lambda(x, Y)\right\|\right)\right\} \\
D \Phi_{0}(x, Y)=\left(\begin{array}{cc}
2 x f^{\prime}\left(x^{2}-\alpha \cdot Y\right) & -\alpha f^{\prime}\left(x^{2}-\alpha \cdot Y\right) \\
0 & \mathbf{0}
\end{array}\right)
\end{gathered}
$$

As we have $2 x f^{\prime}\left(x^{2}\right)=\varphi^{\prime}(x)$ and the derivative of the Feigenbaum map $\varphi$ in dimension one is smaller than $|\lambda|^{-1}$ for $x \in[-\lambda, \lambda]$, we have for $\alpha$ 
small enough that $\left\|D \phi_{0} \circ \Lambda\right\|<a<|\lambda|^{-1}$. Then, $\left\|F_{r}\right\| \leq|\lambda|^{r}\left(a^{r}|\lambda|^{-2}+b\right)$, where $b=\sup _{(x, Y) \in D}\left\|\Lambda^{-1} D \Phi_{0} \circ \Phi_{0} \circ \Lambda(x, Y)\right\|$, proving the lemma.

Remark 2.5. - We can bound $a<(1-\lambda) /(1+\lambda)<2.3312 ; \lambda=$ $-.3995 \ldots ; b<6.2657$. So, $r_{0}$ can be taken equal to 27 .

We recall the proposition 1.5 of the analytic theory in the subsection 1.1. In $\mathcal{H}_{D}$, the spectrum of $D \mathcal{F}\left(\Phi_{0}\right)$ has eigenvalues $1, \lambda^{-1}, \lambda^{-2}$ and $\delta$ with respective spectral invariant finite dimensional subspaces $S_{0}, S_{1}, S_{2}$ and $U$. In particular $\operatorname{dim} U=1$. Let us define $E_{0}, E_{1}, E_{2}$ and $J$ the respective spectral projections in $\mathcal{H}_{D}$. Now we prove that these projections can be extended to $C^{r}$.

LEMMA 2.6. - For $r$ sufficiently large, the projections $E_{0}, E_{1}, E_{2}$ and $J$ defined in $\mathcal{H}_{D}$ can be continuously extended to $C^{r}$, to $C^{r-1}$, and to $C^{r-2}$ as bounded linear operators. Moreover, given $\varepsilon>0$, there exists a positive integer $N$ as large as wanted such that

$$
\left\|F^{N}-\delta^{N} J-E_{0}-\lambda{ }^{N} E_{1}-\lambda^{-2 N} E_{2}\right\| \leq \varepsilon
$$

where the norm $\|\cdot\|$ of the linear operator can be taken either in $C^{r}$, in $C^{r-1}$, or in $C^{r-2}$. The spectrum of $F$ has $1, \lambda^{-1}, \lambda^{-2}$ and $\delta$ as the only eigenvalues with modulus greater or equal to 1 , and the respective spectral invariant subspaces are $S_{0}, S_{1}, S_{2}$, and $U$.

Proof. - Due to the lemma 2.4, for $r$ sufficiently large the spectral radius of $F_{r}, F_{r-1}$ and of $F_{r-2}$ are smaller than 1 . Let us take any $\rho, 0<\rho<1$, bound of these spectral radii. Using the lemma 2.2 with $\varepsilon=\frac{1}{4 c}, c$ defined in the lemma 2.3 , there exists $N>0$ sufficiently large, and $\eta>0$ such that $\left\|F^{N}(u)\right\|_{j, \eta} \leq \frac{\rho^{N}}{4 c}\|u\|_{j}$ for all $u \in C^{j}, j=r-2, r-1, r$. We will work with $j=r$, observing that the proof also works for $j=r-2$ and $j=r-1$.

In $\mathcal{H}_{D}$ we define $Q=F-\delta J-E_{0}-\lambda^{-1} E_{1}-\lambda^{-2} E_{2}$. As $J, E_{0}, E_{1}$ and $E_{2}$ are spectral projections on the eigenspaces $U, S_{0}, S_{1}$ and $S_{2}$ with the eigenvalues of modulus greater or equal to one, we have that, if $N$ is large enough, then $Q^{N}=F^{N}-\delta^{N} . J-F_{0}-\lambda^{-N} F_{1}-\lambda^{-2 N} F_{2}$ has norm in $\mathcal{H}_{D}$ smaller than $\frac{1}{2}$.

The unitary ball of $C^{r}$ is compact in $C^{r-1}$ because the Arzela-Ascoli theorem asserts that any sequence of maps bounded in the $C^{r}$ topology has a convergent subsequence in the $C^{r-1}$ topology. The density of analytic maps in $C^{r}$ allows us to construct, given $\varepsilon>0$, a finite set of analytic maps $f_{1}, \ldots, f_{l}$ in the unitary ball of $C^{r}$ such that the balls in the $C^{r-1}$ topology centered at $f_{1}, \ldots f_{l}$ with radius $\varepsilon>0$ covers the unitary ball of $C^{r}$. In other words, given $u \in C^{r}$ with $\|u\|_{r} \leq 1$ there exists $f_{i_{1}} \in\left\{f_{1}, \ldots, f_{l}\right\}$ 
analytic such that $\left\|f_{i_{1}}\right\|_{r} \leq 1$ and $\left\|u-f_{i_{1}}\right\|_{r-1}<\varepsilon$. Working with $N$ chosen at the beginning of the proof,

$$
\left\|F^{N}\left(u-f_{i_{1}}\right)\right\|_{r-1} \leq M\left\|u-f_{i_{1}}\right\|_{r-1}<M \varepsilon
$$

where $M$ is the norm of $F^{N}$ considered as a linear operator of $C^{r-1}$. Now we have that $\left\|F^{N}\left(u-f_{i_{1}}\right)\right\|_{r, \eta} \leq \frac{\rho^{N}}{4 c}\left\|u-f_{i_{1}}\right\|_{r} \leq \frac{\rho^{N}}{2 c}$ because $\|u\|_{r}$ and $\left\|f_{i_{1}}\right\|_{r}$ are less or equal to 1 . Applying the lemma 2.3:

$$
\left\|F^{N}\left(u-f_{i_{1}}\right)\right\|_{r} \leq 2 c \max \left\{\frac{\rho^{N}}{2 c}, \frac{2}{\eta} M \varepsilon\right\}
$$

For $\varepsilon \leq \eta \rho^{N} /(4 M c)$ we have $\left\|F^{N}\left(u-f_{i_{1}}\right)\right\|_{r} \leq \rho^{N}$. We have proved that given $u \in C^{r}$ with $\|u\|_{r} \leq 1$ it is obtained $f_{i_{1}}$ analytic such that $F^{N}(u)=F^{N}\left(f_{i_{1}}\right)+\rho^{N} u_{1}$ with $\left\|u_{1}\right\|_{r} \leq 1$. Applying to $u_{1}$ the same decomposition we have $F^{2 N}(u)=F^{2 N}\left(f_{i_{1}}\right)+\rho^{N} F^{N}\left(f_{i_{2}}\right)+\rho^{2 N} u_{2}$. After $j$ steps:

$$
F^{N j}(u)=F^{N j}\left(f_{i_{1}}\right)+\rho^{N} F^{N(j-1)}\left(f_{i_{2}}\right)+\ldots+\rho^{N(j-1)} F^{N}\left(f_{i_{j}}\right)+\rho^{N j} u_{j}
$$

with $\left\|u_{j}\right\|_{r} \leq 1$. Now, for an analytic map $f$ we have

$$
F^{N}(f)=Q^{N}(f)+\delta^{N} J(f)+E_{0}(f)+\lambda^{-N} E_{1}(f)+\lambda^{-2 N} E_{2}(f)
$$

Substituting we obtain

$$
F^{N j}(u)=A_{u}+A_{1}+A_{2}+B+C
$$

where

$$
\begin{gathered}
A_{0}=E_{0}\left(f_{i_{1}}\right)+\rho^{N} E_{0}\left(f_{i_{2}}\right)+\ldots+\rho^{N(j-1)} E_{0}\left(f_{i_{j}}\right) \\
A_{1}=\lambda^{-N j} E_{1}\left(f_{i_{1}}\right)+\rho^{N} \lambda^{-N(j-1)} E_{1}\left(f_{i_{2}}\right)+\ldots+\rho^{N(j-1)} \lambda^{-N} E_{1}\left(f_{i_{j}}\right) \\
A_{2}=\lambda^{-2 N j} E_{2}\left(f_{i_{1}}\right)+\rho^{N} \lambda^{-2 N(j-1)} E_{2}\left(f_{i_{2}}\right)+\ldots+\rho^{N(j-1)} \lambda^{-2 N} E_{2}\left(f_{i_{j}}\right) \\
B=Q^{N j}\left(f_{i_{1}}\right)+\rho^{N} Q^{N(j-1)}\left(f_{i_{2}}\right)+\ldots+\rho^{N(j-1)} Q^{N}\left(f_{i_{j}}\right)+\rho^{N j} u_{j} \\
C=\delta^{N j} J\left(f_{i_{1}}\right)+\rho^{N} \delta^{N(j-1)} J\left(f_{i_{2}}\right)+\ldots+\rho^{N(j-1)} \delta^{N} J\left(f_{i_{j}}\right)
\end{gathered}
$$

We will prove that $\tilde{E}_{2}(u)=\lim _{j \rightarrow \infty} \lambda^{2 N j} F^{N j}(u)$ exists in the $C^{r}$ topology. We will see later that $\tilde{E}_{2}$ is the wanted extension of $E_{2}$.

$$
\lambda^{2 N j} F^{N j}(u)=\lambda^{2 N j}\left(A_{0}+A_{1}+A_{2}+B+C\right)
$$

Let us show that all the terms at right converge to 0 with $j \rightarrow \infty$ except $\lambda^{2 N j} A_{2}$. There exists $h>1$ such that for $i=1, \ldots, l,\left\|Q^{N j}\left(f_{i}\right)\right\|_{r} \leq h / 2^{j}$. 
This is because $f_{i} \in \mathcal{H}_{D}$ and the norm of $Q^{N}$ in $\mathcal{H}_{D}$ is smaller than $\frac{1}{2}$. Therefore,

$$
\begin{aligned}
\left\|\lambda^{2 N j} B\right\|_{r} \leq & \lambda^{2 N j}\left(\frac{h}{2^{j}}+\rho^{N} \frac{h}{2^{j-1}}+\ldots+\rho^{N(j-1)} \frac{h}{2}+\rho^{N j} h\right)= \\
& =h\left(\frac{\lambda^{2 N}}{2}\right)^{j} \frac{1-\left(2 \rho^{N}\right)^{(j+1)}}{1-2 \rho^{N}} \rightarrow_{j \rightarrow \infty} 0
\end{aligned}
$$

because $\rho<1$ and $|\lambda|<1$.

As the set of maps where the $f_{i}$ is chosen is finite, take $K$ a constant bounding $\left\|J\left(f_{i}\right)\right\|_{r},\left\|E_{0}\left(f_{i}\right)\right\|_{r},\left\|E_{1}\left(f_{i}\right)\right\|_{r},\left\|E_{2}\left(f_{i}\right)\right\|_{r}$, for $i=1, \ldots, l$. Thus

$$
\begin{aligned}
& \left\|\lambda^{2 N j} C\right\|_{r} \leq\left(\lambda^{2} \delta\right)^{N j}\left(K+\frac{\rho^{N}}{\delta^{N}} K+\ldots+\left(\frac{\rho^{N}}{\delta^{N}}\right)^{j-1} K\right) \\
& \leq\left(\lambda^{2} \delta\right)^{N j} K \frac{1-\left(\rho^{N} / \delta^{N}\right)^{j}}{1-\rho^{N} / \delta^{N}} \rightarrow_{j \rightarrow \infty} 0
\end{aligned}
$$

because $\lambda^{2} \delta<1$ and $\rho<1<\delta$

$$
\begin{gathered}
\left\|\lambda^{2 N j} A_{0}\right\|_{r} \leq \lambda^{2 N j}\left(K+\rho^{N} K+\ldots+\rho^{N(j-1)} K\right) \\
\leq K \lambda^{2 N j} \frac{1-\rho^{N j}}{1-\rho^{N}} \rightarrow_{j \rightarrow \infty} 0 \\
\left\|\lambda^{2 N j} A_{1}\right\|_{r} \leq|\lambda|^{N j}\left(K+(\rho|\lambda|)^{N} K+\ldots+(\rho|\lambda|)^{N(j-1)} K\right) \\
\leq K|\lambda|^{N j} \frac{1-\left(\rho^{N}|\lambda|^{N}\right)^{j}}{1-\rho^{N}|\lambda|^{N}} \rightarrow_{j \rightarrow \infty} 0 \\
\lambda^{2 N_{j}} A_{2}=E_{2}\left(f_{i_{1}}\right)+\rho^{N} \lambda^{2 N} E_{2}\left(f_{i_{2}}\right)+\ldots+\rho^{N(j-1)} \lambda^{2 N(j-1)} E_{2}\left(f_{i_{j}}\right)
\end{gathered}
$$

This series is majored by

$$
\left\|\lambda^{2 N j} A_{2}\right\|_{r} \leq K+\rho^{N} \lambda^{2 N} K+\ldots+\rho^{N(j-1)} \lambda^{2 N(j-1)} K \leq K \frac{1-\left(\rho^{N} \lambda^{2 N}\right)^{j}}{1-\rho^{N} \lambda^{2 N}}
$$

which is convergent because $\rho<1$ and $\lambda<1$. Then, we define

$$
\tilde{E}_{2}(u)=\lim _{j \rightarrow \infty} \lambda^{2 N j} F^{N j}(u)=\lim _{j \rightarrow \infty} \lambda^{2 N j} A_{2}=\sum_{k=0}^{\infty}\left(\rho^{N} \lambda^{2 N}\right)^{k} E_{2}\left(f_{i_{k+1}}\right)
$$

It is clear that $\tilde{E}_{2}(u)$ is an analytic function because $S_{2}$ is closed and formed by analytic functions ( $S_{2}$ is a finite dimensional space). 
Then, we define $\tilde{J}$, (which will be the extension of $J$, corresponding to the eigenvalue $\delta$ ). We apply the same method to show that $\tilde{J}(u)=\lim _{j \rightarrow \infty} \delta^{-N j}\left(F^{N j}(u)-\lambda^{-2 N j} \tilde{E}_{2}(u)\right)$ exists. We must study $\delta^{-N j}\left(A_{2}-\lambda^{-2 N j} \dot{E}_{2}(u)\right)+\delta^{-N j}\left(B+C+A_{0}+A_{1}\right)$. The first term is

$$
\begin{gathered}
\delta^{-N j} \lambda^{-2 N j}\left(\sum_{k=0}^{j-1}\left(\rho^{N} \lambda^{2 N}\right)^{k} E_{2}\left(f_{i_{k+1}}\right)-\sum_{k=0}^{\infty}\left(\rho^{N} \lambda^{2 N}\right)^{k} E_{2}\left(f_{i_{k+1}}\right)\right)= \\
=-\delta^{-N j} \lambda^{-2 N j} \sum_{k=j}^{\infty}\left(\rho^{N} \lambda^{2 N}\right)^{k} E_{2}\left(f_{i_{k+1}}\right)
\end{gathered}
$$

Thus, the $r$-norm of the first term is bounded by

$$
\delta^{-N j} \lambda^{-2 N j} K \sum_{k=j}^{\infty}\left(\rho^{N} \lambda^{2 N}\right)^{k}=K \delta^{-N j} \lambda^{-2 N j} \frac{\left(\rho^{N} \lambda^{2 N}\right)^{j}}{1-\rho^{N} \lambda^{2 N}} \rightarrow_{j \rightarrow \infty} 0
$$

because $\rho \delta^{-1}<1$.

The second term is decomposed. $\left\|\delta^{-N j} B\right\|_{r}$ is treated as $\lambda^{2 N j} B$ above, substituting $\lambda^{2}$ by $\delta^{-1}$, proving $\left\|\delta^{-N j} B\right\|_{r} \rightarrow_{j \rightarrow \infty} 0$.

Analogously are treated $\left\|\delta^{-N j} A_{0}\right\|_{r}$ and $\left\|\delta^{-N_{j}} A_{1}\right\|_{r} \rightarrow_{j \rightarrow \infty} 0$. Now,

$$
\begin{aligned}
& \left\|\delta^{-N j} C\right\|_{r} \leq\left\|J\left(f_{i_{1}}\right)\right\|_{r}+\rho^{N} \delta^{-N}\left\|J\left(f_{i_{2}}\right)\right\|_{r} \\
& +\ldots+\left(\rho^{N} \delta^{-N}\right)^{j-1}\left\|J\left(f_{i_{j}}\right)\right\|_{r} \leq K \sum_{k=0}^{\infty}\left(\rho^{N} \delta^{-N}\right)^{k}
\end{aligned}
$$

Thus $\tilde{J}(u)=\sum_{k=0}^{\infty}\left(\rho^{N} \delta^{-N}\right)^{k} J\left(f_{i_{k+1}}\right)$. As before, $\tilde{J}(u)$ is analytic.

With the same procedure we define

$$
\begin{gathered}
\tilde{E}_{1}(u)=\lim _{j \rightarrow \infty} \lambda^{N j}\left(F^{N j}(u)-\lambda^{-2 N j} \tilde{E}_{2}(u)-\delta^{N j} \tilde{J}(u)\right) \\
=\sum_{k=0}^{\infty}\left(\rho^{N} \lambda^{N}\right)^{k} E_{1}\left(f_{i_{k+1}}\right) \\
\tilde{E}_{0}(u)=\lim _{j \rightarrow \infty} F^{N j}(u)-\lambda^{-2 N j} \tilde{E}_{2}(u)-\delta^{N j} \tilde{J}(u)-\lambda^{-N j} \tilde{E}_{1}(u) \\
=\sum_{k=0}^{\infty} \rho^{N k} E_{0}\left(f_{i_{k+1}}\right)
\end{gathered}
$$

$\tilde{E}_{1} u$ and $\tilde{E}_{0} u$ are analytic functions. 
We have

$$
\begin{gathered}
\left\|F^{N j}(u)-\lambda^{-2 N j} \tilde{E}_{2}(u)-\delta^{N j} \tilde{J}(u)-\lambda^{-N j} \tilde{E}_{1}(u)-\tilde{E}_{0}(u)\right\|_{r} \leq \\
\leq K \lambda^{-2 N j} \sum_{k=j}^{\infty}\left(\rho^{N} \lambda^{2 N}\right)^{k}+K \delta^{N j} \sum_{k=j}^{\infty}\left(\rho^{N} \delta^{-N}\right)^{k}+ \\
+K|\lambda|^{-N j} \sum_{k=j}^{\infty}\left(\rho^{N}|\lambda|^{N}\right)^{k}+K \sum_{k=j}^{\infty} \rho^{N k}+\frac{h}{2^{j}} \sum_{k=0}^{j}\left(2 \rho^{N}\right)^{k}<\varepsilon
\end{gathered}
$$

for $j$ large enough uniformly for all $u$ with $\|u\|_{r} \leq 1$. We know that $\tilde{E}_{i}\left(C^{r}\right) \subset S_{i}$ for $i=0,1,2$, and $\tilde{J}\left(C^{r}\right) \subset U$. In particular, if $u$ is analytic, from (6) we conclude that $\tilde{E}_{i}(u)=E_{i}(u), i=0,1,2$ and $\tilde{J}(u)=J(u)$. The formula (6) ends the proof of the lemma.

From now on we will take $r$ sufficiently large to apply the lemma 2.6.

The projection $J$ on the one-dimensional space $U$ of the eigenvectors with eigenvalue $\delta$ can be written as

$$
J(u)=a(u) v
$$

where $v=\left.\frac{d}{d t} \Phi_{t}\right|_{t=0} \in U$ and $a(u) \in \mathbf{R}$ for all $u \in C^{j}, j=r, r-1, r-2$; $a$ is linear and bounded.

The sum of the eigenspaces of $F$ corresponding to the eigenvalues 1 , $\lambda^{-1}$, and $\lambda^{-2}$ is $\bar{S}=S_{0} \oplus S_{1} \oplus S_{2}$. The same proof of the proposition 1.5, part $c$ ) shows that for any $u \in C^{r}$ there exists $\sigma[u]$, the unique analytic map in $\mathbf{C}^{n}$ such that $\psi_{\sigma[u]}=E u \in \bar{S}$, where $E=E_{0} \oplus E_{1} \oplus E_{2}$. The transformation $u \mapsto \sigma[u]$ is linear and bounded. We remark that $\operatorname{Im} \sigma$ is a finite dimensional space. For $G \in C^{r}$ in a neighborhood $\mathcal{U}_{i}$ of $\Phi_{0}$, we define

$$
T_{i}(G)=\left(I-\sigma\left[\mathcal{F}^{i}(G)-\Phi_{0}\right]\right)^{-1} \circ \mathcal{F}^{i}(G) \circ\left(I-\sigma\left[\mathcal{F}^{i}(G)-\Phi_{0}\right]\right)
$$

where $\mathcal{F}^{i}$ was defined in (2) at the beginning of this section. When restricted to $\mathcal{H}_{D}$ the transformation $T_{i}$ and $\mathcal{F}^{i}$ are Fréchet differentiable. Its derivatives at $\Phi_{0}$ can be extended to $C^{r}, r \geq 1$ as bounded linear operators $\tilde{F}_{i}$ and $F_{i}$ respectively, which will be called the formal derivatives at $\Phi_{0}$. Let us observe that $F_{i}=d \mathcal{F}^{i}\left(\Phi_{0}\right)=\left(d \mathcal{F}\left(\Phi_{0}\right)\right)^{i}=F^{i}$. We also have $\tilde{F}_{i} \cdot u=d T_{i}\left(\Phi_{0}\right) \cdot u=F^{i} u+\sigma\left[F^{i} u\right] \circ \Phi_{0}-D \Phi_{0} \cdot \sigma\left[F^{i} u\right]=(I-E)\left(F^{i} \cdot u\right)$.

Lemma 2.7. - Given $\varepsilon>0$, there exists $N$ as large as wanted such that

$$
\left\|\tilde{F}_{N}-\delta^{N} J\right\| \leq \varepsilon
$$

where the norm $\|\cdot\|$ of the linear operator can be taken either in $C^{r}$, in $C^{r-1}$, or in $C^{r-2}$ 
Proof. - We will make explicit computations in $C^{r}$, but the same argument is valid in $C^{r-1}$ and $C^{r-2}$. Using the density of $\mathcal{H}_{D}$ in $C^{r}$ it can be seen that $E_{0}, E_{1}, E_{2}$ and $J$ commute with $F$ in $C^{r}$. Thus

$$
\begin{aligned}
& \left\|\tilde{F}_{N} \cdot u-\delta^{N} J(u)\right\|_{r}=\left\|(I-E) F^{N} \cdot u-\delta^{N} J(u)\right\|_{r} \\
& \quad=\left\|F^{N} \cdot u-E F^{N} \cdot u-\delta^{N} J(u)\right\|_{r} \\
& \quad=\left\|F^{N} \cdot u-E_{0}(u)-\lambda^{-N} E_{1}(u)-\lambda^{-2 N} E_{2}(u)-\delta^{N} J(u)\right\|_{r} \leq \varepsilon\|u\|_{r}
\end{aligned}
$$

The last inequality is due to the lemma 2.6 .

Now, let $\left\{b_{i}\right\}_{i=0, \ldots 2^{N}-1}$ be a finite set of maps in $C^{1}$ with $b_{i}(D) \subset D$. Let $\left\{a_{i}\right\}_{i=0, \ldots 2^{N}-1}$ be a finite set of matrices $n \times n$ depending $C^{0}$ of $x \in D$. We define a bounded linear operator on $C^{0}\left(D, \mathcal{L}_{r}\left(\mathbf{R}^{n}, \mathbf{R}^{n}\right)\right)$ by

$$
\Omega H(x)=\sum_{i=0}^{2^{N}-1} a_{i}(x) \cdot b_{i}^{*} H(x)
$$

Let

$$
M=\sup _{x \in D} \sum_{i=0}^{2^{N}-1}\left\|a_{i}(x)\right\|\left\|D b_{i}(x)\right\|^{r}
$$

Then, we have

LEMmA 2.8. - Given $\varepsilon>0$, there exists $\zeta>0$ such that if $\tilde{a}_{i}(x) \in$ $\mathcal{L}\left(\mathbf{R}^{n}, \mathbf{R}^{n}\right)$ depending $C^{0}$ on $x \in D$, and $\tilde{b}_{i} \in C^{1}$ with $\left\|b_{i}-\tilde{b}_{i}\right\|_{1}<\zeta$, $\left\|a_{i}-\tilde{a}_{i}\right\|_{0}<\zeta$ and $\tilde{b}_{i}(D) \subset D$ for $i=0, \ldots 2^{N}-1$, then the corresponding operator $\tilde{\Omega}$ on $C^{0}\left(D, \mathcal{L}_{r}\left(\mathbf{R}^{n}, \mathbf{R}^{n}\right)\right)$ satisfies $\|\tilde{\Omega}-\Omega\|<2(M+\varepsilon)$.

Proof.

$$
\Omega H-\Omega H=\sum_{i=0}^{2^{N}-1} a_{i}\left[b_{i}^{*} H-\tilde{b}_{i}^{*} H\right]+\sum_{i=0}^{2^{N}-1}\left[a_{i}-\tilde{a}_{i}\right] \tilde{b}_{i}^{*} H
$$

The second term has $C^{0}$ norm bounded by $\sum_{i=0}^{2^{N}-1} K\|H\|_{0}\left\|a_{i}-\tilde{a}_{1}\right\|_{0}$ for some constant $K$. Taking $\zeta$ small enough this term is smaller than $\varepsilon\|H\|_{0}$. The first term can be written as

$$
\begin{aligned}
\sum_{i=0}^{2^{N}-1} a_{i}\left[H \circ b_{i}-H \circ \tilde{b}_{i}\right] \cdot\left[D b_{i} \cdot, \ldots, D b_{i} \cdot\right] \\
+\sum_{i=0}^{2^{N}-1} a_{i} H \circ \tilde{b}_{i} \cdot\left[\left(D b_{i}-D \tilde{b}_{i}\right) \cdot, \ldots, D b_{i} \cdot\right]+\ldots+ \\
+\sum_{i=0}^{2^{N}-1} a_{i} H \circ \tilde{b}_{i} \cdot\left[D \tilde{b}_{i} \cdot \ldots,\left(D b_{i}-D \tilde{b}_{i}\right) \cdot\right]
\end{aligned}
$$


As $\left\|b_{i}-\tilde{b}_{i}\right\|_{1}<\zeta$ the last terms of this sum can be treated as the former second term and proved to have $C^{0}$ norm smaller than $\varepsilon\|H\|_{0}$. The first term of this sum evaluated at a point $x \in D$ is in $\mathcal{L}_{r}\left(R^{n}, R^{n}\right)$ and has norm bounded by

$$
\sum_{i=0}^{2^{N}-1}\left\|a_{i}(x)\right\|\left\|D b_{i}(x)\right\|^{r} 2\|H\|_{0} \leq 2 M\|H\|_{0}
$$

Hence, the lemma is proved.

LemMA 2.9. - For all $N>0$ large enough there exists $\zeta>0$ so that if $t \in \mathbf{R}$ and $u \in C^{r}$ with $|t|<\zeta$ and $\|u\|_{r}<\zeta$, then $\left\|T_{N}\left(\Phi_{t}+u\right)-T_{N}\left(\Phi_{t}\right)-\tilde{F}_{N} \cdot u\right\|_{r} \leq \frac{1}{8}\|u\|_{r}$.

Proof. - For given $\varepsilon>0$, let us take $N$ such that $\left(F_{r}\right)^{n}$ has norm less than $\varepsilon$ as an operator on $C^{0}$ (lemma 24). After some computations we find that the functional derivative of $T_{N}$ at a point $G \in \mathcal{H}_{D}$, considered in the set of analytic functions is

$$
\left.\begin{array}{c}
d T_{N}(G) \cdot u=\left(I-D \sigma\left[\mathcal{F}^{N}(G)-\Phi_{0}\right] \circ\left(I-\sigma\left[\mathcal{F}^{N}(G)-\Phi_{0}\right]\right)^{1}\right. \\
\left.\circ \mathcal{F}^{N}(G) \circ\left(I-\sigma\left[\mathcal{F}^{N}(G)-\Phi_{0}\right]\right)\right)^{-1} \\
\cdot\left\{\left[\Lambda_{N, G}^{-1}\left(\sum_{k=1}^{2^{N}}\left(\prod_{s=1}^{k-1} D G\left(G^{2^{N}-s} \circ \Lambda_{N, G}\right)\right) \cdot u\left(G^{2^{N}-k} \circ \Lambda_{N, G}\right)\right)+\text { o.t. }\right]\right. \\
\circ\left(I-\sigma\left[\mathcal{F}^{N}(G)-\Phi_{0}\right]\right)+ \\
+\sigma\left[\Lambda_{N, G}^{-1}\left(\sum_{k=1}^{2^{N}}\left(\prod_{s=1}^{k-1} D G\left(G^{2^{N}-s} \circ \Lambda_{N, G}\right)\right) \cdot u\left(G^{2^{N}-k} \circ \Lambda_{N, G}\right)\right)+\text { o.t. }\right] \\
\circ\left(I-\sigma\left[\mathcal{F}^{N}(G)-\Phi_{0}\right]\right)^{-1} \circ \mathcal{F}^{N}(G) \circ\left(I-\sigma\left[\mathcal{F}^{N}(G)-\Phi_{0}\right]\right)- \\
\cdot \sigma\left[\mathcal{F}^{N}(G) \circ\left(I-\sigma\left[\mathcal{F}^{N}(G)-\Phi_{0}\right]\right) \cdot\right. \\
\left.\left[\Lambda_{N, G}^{-1}\left(\sum_{k=1}^{2^{N}}\left(\prod_{s=1}^{k-1} D G\left(G^{2 N-s} \circ \Lambda_{N, G}\right)\right) \cdot u\left(G^{2^{N}-k} \circ \Lambda_{N, G}\right)\right)+\text { o.t. }\right]\right\}
\end{array}\right\}
$$

where o.t. represents terms in which $u$ appears evaluated in some point of the space. If $G$ is analytic $d T_{N}(G)$ can be extended to a bounded linear operator in $C^{r}$. We recall that $\operatorname{Im} \sigma \subset \mathcal{H}_{D}$ and is finite dimensional. 
Let us define a bounded linear operator $\mathcal{A}(G)$ on $C^{0}\left(D, \mathcal{L}_{r}\left(\mathbf{R}^{n}, \mathbf{R}^{n}\right)\right)$ as

$$
\begin{gathered}
\mathcal{A}(G) \cdot w=\left(I-D \sigma\left[\mathcal{F}^{N}(G)-\Phi_{0}\right] \circ\left(I-\sigma\left[\mathcal{F}^{N}(G)-\Phi_{0}\right]\right)^{-1}\right. \\
\left.\circ \mathcal{F}^{N}(G) \circ\left(I-\sigma\left[\mathcal{F}^{N}(G)-\Phi_{0}\right]\right)\right)^{-1} . \\
\left\{\Lambda _ { N , G } ^ { - 1 } \left(\sum_{k=1}^{2^{N}}\left(\prod_{s=1}^{k-1} D G\left(G^{2^{N}-s} \circ \Lambda_{N, G} \circ\left(I-\sigma\left[\mathcal{F}^{N}(G)-\Phi_{0}\right]\right)\right)\right)\right.\right. \\
\left.\left.\left(\left(G^{2^{N}-k} \circ \Lambda_{N, G} \circ\left(I-\sigma\left[\mathcal{F}^{N}(G)-\Phi_{0}\right]\right)\right)^{*} w\right)\right)\right\}
\end{gathered}
$$

If $G$ is analytic and $u \in C^{r}$, we define $\mathcal{B}, \mathcal{C}, \ldots, \mathcal{M}$ as

$D^{r}\left(d T_{N}(G) \cdot u\right)=\mathcal{A}(G) \cdot D^{r} u+\mathcal{B}(G) \cdot D^{r-1} u+\mathcal{C}(G) \cdot D^{r-2} u+\ldots+\mathcal{M}(G) \cdot u$ depending nonlinearly on $G$ and its first $r+1$ derivatives.

Let us compute the spatial derivatives of $T_{N}(G)$ for $G \in C^{r}$.

$$
\begin{gathered}
D T_{N}(G)=\left(I-D \sigma\left[\mathcal{F}^{N}(G)-\Phi_{0}\right] \circ\left(I-\sigma\left[\mathcal{F}^{N}(G)-\Phi_{0}\right]\right)^{-1}\right. \\
\left.\circ \mathcal{F}^{N}(G) \circ\left(I-\sigma\left[\mathcal{F}^{N}(G)-\Phi_{0}\right]\right)\right)^{-1} \cdot \\
\cdot \Lambda_{N, G}^{-1} \prod_{k=1}^{2^{N}} D G\left(G^{2^{N}-k} \circ \Lambda_{N, G} \circ\left(I-\sigma\left[\mathcal{F}^{N}(G)-\Phi_{0}\right]\right)\right) \\
\times \Lambda_{N, G}\left(I-D \sigma\left[\mathcal{F}^{N}(G)-\Phi_{0}\right]\right)
\end{gathered}
$$

When computing the spatial derivative of $T_{N}(G)$ of order $r \geq 2$ we are interested into separating the terms depending on $D^{r} G$. In $D^{2} T_{N}(G)$ the term having $D^{2} G$ is

$$
\begin{gathered}
\left(I-D \sigma\left[\mathcal{F}^{N}(G)-\Phi_{0}\right] \circ\left(I-\sigma\left[\mathcal{F}^{N}(G)-\Phi_{0}\right]\right)^{-1}\right. \\
\left.\circ \mathcal{F}^{N}(G) \circ\left(I-\sigma\left[\mathcal{F}^{N}(G)-\Phi_{0}\right]\right)\right)^{-1} \\
\cdot \Lambda_{N, G}^{-1} \sum_{k=1}^{2^{N}}\left(\prod_{s=1}^{k-1} D G\left(G^{2^{N}-s} \circ \Lambda_{N, G} \circ\left(I-\sigma\left[\mathcal{F}^{N}(G)-\Phi_{0}\right]\right)\right)\right) . \\
\cdot\left(G^{2^{N}-k} \circ \Lambda_{N, G} \circ\left(I-\sigma\left[\mathcal{F}^{N}(G)-\Phi_{0}\right]\right)\right)^{*} D^{2} G
\end{gathered}
$$

In general for $r \geq 2, D^{r} T_{N}(G)$ has a term depending on $D^{r} G$ that is $\mathcal{A}(G) \cdot D^{r}(G)$. 
Take $u \in C^{r}$. When computing $D^{r}\left(T_{N}\left(\Phi_{t}+u\right)-T_{N}\left(\Phi_{t}\right)\right)$ we obtain a term depending on $D^{r} u$ that is precisely $\mathcal{A}\left(\Phi_{t}+u\right) D^{r} u$, plus other term $\mathcal{P}\left(\Phi_{t}, u\right)$ depending on $\Phi_{t}$ and its first $r$ derivatives and on $u$ and its first $r-1$ derivatives. It is a sum of compositions and multiplications of these maps. Thus $\mathcal{P}\left(\Phi_{t}, \cdot\right)$ is a transformation from $C^{r}$ to $C^{1}$ that is null for $u=0$ and has at $u=0$ Fréchet derivative when looked from $C^{r}$ to $C^{0}$. That is

$$
\mathcal{P}\left(\Phi_{t}, u\right)=P\left(\Phi_{t}\right) u+N\left(\Phi_{t}, u\right)
$$

with $\left\|N\left(\Phi_{t}, u\right)\right\|_{0} \leq \varepsilon\|u\|_{r}$ if $\|u\|_{r}$ is sufficiently small.

$P\left(\Phi_{t}\right) u$ is linear on $u$ and its first $r-1$ derivatives. It is the linear part on $u$ obtained from $D^{r}\left(T_{N}\left(\Phi_{t}+u\right)-T_{N}\left(\Phi_{t}\right)\right)$ taking away the term $\mathcal{A}\left(\Phi_{t}+u\right) D^{r} u$. As

$$
D^{r}\left(d T_{N}\left(\Phi_{t}\right) u\right)=\mathcal{A}\left(\Phi_{t}\right) D^{r} u+\mathcal{B}\left(\Phi_{t}\right) D^{r-1} u+\ldots+\mathcal{M}\left(\Phi_{t}\right) u
$$

we have

$$
P\left(\Phi_{t}\right) u=\mathcal{B}\left(\Phi_{t}\right) D^{r-1} u+\ldots+\mathcal{M}\left(\Phi_{t}\right) u
$$

Thus

$$
\begin{aligned}
D^{r}\left(T_{N}\left(\Phi_{t}+u\right)-T_{N}\left(\Phi_{t}\right)\right)=\mathcal{A}\left(\Phi_{t}+u\right) D^{r} u+\mathcal{B}\left(\Phi_{t}\right) D^{r-1} u \\
+\mathcal{C}\left(\Phi_{t}\right) D^{r-2} u+\ldots+\mathcal{M}\left(\Phi_{t}\right) u+N\left(\Phi_{t}, u\right)
\end{aligned}
$$

where $\left\|N\left(\Phi_{t}, u\right)\right\|_{0} \leq \varepsilon\|u\|_{r}$ for any given $\varepsilon$ if $\|u\|_{r}$ is sufficiently small.

Provided that $|t|$ is small enough,

$$
\begin{gathered}
\left\|\left(\mathcal{B}\left(\Phi_{t}\right)-\mathcal{B}\left(\Phi_{0}\right)\right) \cdot D^{r-1} u\right\|_{0}, \\
\left\|\left(\mathcal{C}\left(\Phi_{t}\right)-\mathcal{C}\left(\Phi_{0}\right)\right) \cdot D^{r-2} u\right\|_{0}, \ldots,\left\|\left(\mathcal{M}\left(\Phi_{t}\right)-\mathcal{M}\left(\Phi_{0}\right)\right) \cdot u\right\|_{0}
\end{gathered}
$$

will be all less that $\frac{\varepsilon}{r}\|u\|_{r}$.

We must study $\mathcal{A}(G)$. We observe that

$$
\mathcal{A}\left(\Phi_{0}\right)=\Lambda_{N, \Phi_{0}}^{-1}\left(\sum_{k=1}^{2^{N}}\left(\prod_{s=1}^{k-1} D \Phi_{0}\left(\Phi_{0}^{2^{N}-s} \circ \Lambda_{N, \Phi_{0}}\right)\right)\left(\Phi_{0}^{2^{N}-k} \circ \Lambda_{N, \Phi_{0}}\right)^{*}\right)
$$

Now, we apply the lemma 2.8 to $\mathcal{A}\left(\Phi_{0}\right)$, the corresponding $M$ is $\left\|\left(F^{N}\right)_{r}\right\| \leq\left\|\left(F_{r}\right)^{N}\right\|<\varepsilon$. We conclude that if $|t|$ and $\|u\|_{r}$ are small 
enough, then $\mathcal{A}\left(\Phi_{t}+u\right)-\mathcal{A}\left(\Phi_{0}\right)$ has norm less than $4 \varepsilon$ as an operator on $C^{0}$. Putting everything together, and noting that

$$
D^{r}\left(\tilde{F}_{N} \cdot u\right)=\mathcal{A}\left(\Phi_{0}\right) \cdot D^{r} u+\mathcal{B}\left(\Phi_{0}\right) \cdot D^{r-1} u+\ldots+\mathcal{M}\left(\Phi_{0}\right) \cdot u
$$

we obtain

$$
\left\|D^{r}\left(T_{N}\left(\Phi_{t}+u\right)-T_{N}\left(\Phi_{t}\right)-\tilde{F}_{N} \cdot u\right)\right\|_{0} \leq 6 \varepsilon\|u\|_{r}
$$

$T_{N}$ as a transformation from $C^{r}$ to $C^{r-1}$ is differentiable Fréchet, so, for $|t|$ and $\|u\|_{r}$ small enough:

$$
\left\|T_{N}\left(\Phi_{t}+u\right)-T_{N}\left(\Phi_{t}\right)-\tilde{F}_{N} \cdot u\right\|_{r-1} \leq \varepsilon\|u\|_{r}
$$

If $\varepsilon \leq \frac{1}{48}$ :

$$
\left\|T_{N}\left(\Phi_{t}+u\right)-T_{N}\left(\Phi_{t}\right)-\tilde{F}_{N} \cdot u\right\|_{r} \leq \frac{1}{8}\|u\|_{r}
$$

Remark 2.10. - At the beginning of the proof of the lemma 2.9, we have computed the derivative $d T_{N}(G)$ for $G \in \mathcal{H}_{D}$. The right term of the equality (7) defines for any $G$ of class $C^{r}$ a bounded linear operator in $C^{r-1}$ and also in $C^{r-2}$. This operator will be called the formal derivative of $T_{N}$ at $G$. We do not have continuous dependence of this operator at $G \in C^{r}$. But, arguing as in the proof of the former lemma and using the lemma 2.8 with $M \leq\left\|\left(F_{r}\right)^{N}\right\|$ we obtain the following result:

Given $\varepsilon>0$ there exists $N$ large enough and $\zeta>0$ such that if $\left\|G-\Phi_{0}\right\|_{r}<\zeta$, then $d T_{N}(G)-d T_{N}\left(\Phi_{0}\right)$ has norm less than $\varepsilon$ as linear operator on $C^{r-1}$.

Proof of the proposition. 2.1. - We apply the lemmas 2.7 and 2.9 to obtain

$$
\left\|T_{N}\left(\Phi_{t}+u\right)-T_{N}\left(\Phi_{t}\right)-\delta^{N} J(u)\right\|_{r} \leq \frac{1}{4}\|u\|_{r}
$$

We have $T_{N}\left(\Phi_{t}\right)=\Phi_{\delta^{N} t}$ and $J(u)=a(u) v$ where $v=\left.\frac{d}{d s} \Phi_{s}\right|_{s=0}$. So

$$
\left\|T_{N}\left(\Phi_{t}+u\right)-\Phi_{\delta^{N} t}-\delta^{N} a(u) v\right\|_{r} \leq \frac{1}{4}\|u\|_{r}
$$

for $\Phi_{t}$ is a curve of class $C^{1}$ of maps in $C^{r}$, thus $\Phi_{\delta^{N}(t+a(u))}-\Phi_{\delta^{N} t}=$ $\left.\frac{d}{d s} \Phi_{s}\right|_{s=\delta^{N} t} \delta^{N} a(u)+\Delta$ where $\|\Delta\|_{r} \leq \frac{1}{8}\|u\|_{r}$ if $\|u\|_{r}$ and $|t|$ are small enough. Moreover if $|t|$ is small then

$$
\left\|\left.\frac{d \Phi_{s}}{d s}\right|_{s=\delta^{N} t}-v\right\|_{r} \delta^{N}|a(u)| \leq \frac{1}{8}\|u\|_{r}
$$

We obtain

$$
\left\|\Phi_{\delta^{N}(t+a(u))}-\Phi_{\delta^{N} t}-\delta^{N} a(u) v\right\|_{r} \leq \frac{1}{4}\|u\|_{r}
$$

and we deduce that $\left\|T_{N}\left(\Phi_{t}+u\right)-\Phi_{\delta^{N}(t+a(u))}\right\|_{r} \leq \frac{1}{2}\|u\|_{r}$, as wanted. 


\section{THE STABLE MANIFOLD}

In this section we prove the existence of the stable manifold of the renormalization in the space $C^{r}$ and deduce the theorem 2 .

We recall that the projection $J$ on the one-dimensional subspace $U$ corresponding to the eigenvalue $\delta$ is written as $J(u)=a(u) v$, where $v=\left.\frac{d}{d t} \Phi_{t}\right|_{t=0} \in U$ and $a$ is linear and bounded from $C^{r}, C^{r-1}$ or $C^{r-2}$ to R. Thus $a(v)=1$ and $\left.\frac{d}{d t} a\left(\Phi_{t}-\Phi_{0}\right)\right|_{t=0}=a(v)=1$. We denote $\|a\|_{r}$ the norm of $a$ as a linear operator from $C^{r}$ to $\mathbf{R}$. Analogously $\|a\|_{r-1},\|a\|_{r-2}$.

Let $\zeta$ as in the proposition 2.1 and also verifying that $\left|a\left(\Phi_{t}-\Phi_{0}\right)-t\right| \leq$ $|t| / 4$ for $|t|<\zeta$.

Now we are ready to define the stable set for $T_{N}$. Choose positive numbers $\varepsilon$ and $\varepsilon^{\prime}$ smaller than one so that

$$
\begin{gathered}
2 \delta^{N} \varepsilon^{\prime}<\zeta \\
\varepsilon \max \left(1,30\|a\|_{r}\right)<\varepsilon^{\prime}
\end{gathered}
$$

Define the set $W=\left\{\Phi_{0}+u, u \in C^{r},\|u\|_{r}<\varepsilon\right\}$. Given $\Phi_{0}+u \in W$, we define recursively the finite or infinite sequences $\left\{t_{k}\right\}_{k \geq 0}$ and $\left\{u_{k}\right\}_{k \geq 0}$ by the relations

$$
\begin{gathered}
t_{0}=0 ; u_{0}=u \\
T_{N}^{k}\left(\Phi_{0}+u\right)=\Phi_{t_{k}}+u_{k} \\
t_{k+1}=\delta^{N}\left(t_{k}+a\left(u_{k}\right)\right) \text { if }\left|t_{k}\right|<\zeta
\end{gathered}
$$

They are defined as long as $\left|t_{k}\right|<\zeta$. By the proposition 2.1 we have $\left\|u_{k}\right\|_{r}<2^{-k} \varepsilon$. Moreover, if $\left|t_{k}\right|<\varepsilon^{\prime}$, then

$$
\left|t_{k+1}\right| \leq \delta^{N}\left(\varepsilon^{\prime}+\|a\|_{r} \varepsilon\right) \leq 2 \delta^{N} \varepsilon^{\prime}<\zeta
$$

There are three mutually exclusive possibilities:

i) for some $k, \varepsilon^{\prime}<t_{k}<\zeta$ and $\left|t_{j}\right| \leq \varepsilon^{\prime}$ for $j<k$.

ii) for some $k, \varepsilon^{\prime}<-t_{k}<\zeta$ and $\left|t_{j}\right| \leq \varepsilon^{\prime}$ for $j<k$.

iii) $\left|t_{k}\right| \leq \varepsilon^{\prime}$ for all $k \geq 0$.

We denote $W_{+}, W_{-}$and $W_{0}$ the subsets of $W$ where respectively i), ii) or iii) holds. The map $u \mapsto t_{k}$ is continuous on $W$, so $W_{+}$and $W_{-}$are open. Moreover $\Phi_{t} \in W_{+}\left(W_{-}\right)$for all $t>0$ (resp. $t<0$ ) small enough. Hence $W_{0}$ is a relatively closed subset of $W$ disconnecting it. 
If $\Phi_{0}+u \in W_{0}$ then there are infinite sequences $\left\{t_{k}\right\}_{k \geq 0}$ and $\left\{u_{k}\right\}_{k \geq 0}$. We assert that

$$
\left|t_{k}\right|<\varepsilon\|a\|_{r} 2^{-k+1}
$$

In fact, suppose that there exists $k$ such that $\left|t_{k}\right| \geq \varepsilon\|a\|_{r} 2^{-k+1}$. As $t_{k+1}=$ $\delta^{N}\left(t_{k}+a\left(u_{k}\right)\right)$ and $\left\|u_{k}\right\|_{r}<2^{-k} \varepsilon$, we obtain $\left|t_{k+1}\right| \geq \delta^{N}\|a\|_{r} \varepsilon 2^{-k} \geq$ $\varepsilon\|a\|_{r} 2^{-k+2}$. Repeating the argument $\left|t_{k+j}\right| \geq \varepsilon\|a\|_{r} 2^{-k+j+1}$ for all $j \geq \overline{0}$, and this contradicts the inequality $\left|t_{k+j}\right| \leq \varepsilon^{\prime}$ for all $j$.

Therefore, for $\Phi_{0}+u \in W_{0}, T_{N}^{k}\left(\Phi_{0}+u\right) \rightarrow \Phi_{0}$ as $k \rightarrow \infty$ and $\left\|T_{N}^{k}\left(\Phi_{0}+u\right)-\Phi_{0}\right\|_{r}=O\left(2^{-k}\right) . W_{0}$ is thus the local stable set for $T_{N}$. To prove that $W_{0}$ is locally a $C^{1}$ submanifold of codimension one we need some previous lemmas, obtained from [4].

Lemma 3.1. - For $G_{1} \in W_{+} \cup W_{-}$, if $\varepsilon^{\prime}<\left|t_{k}\right|<\zeta$ then $\left|a\left(T_{N}^{k} G_{1}-\Phi_{0}\right)\right| \geq \varepsilon^{\prime} / 4$

Proof.

$$
\begin{gathered}
\left|a\left(T_{N}^{k} G_{1}-\Phi_{0}\right)\right|=\left|a\left(\Phi_{\iota_{k}}-\Phi_{0}\right)+a\left(u_{k}\right)\right| \geq\left|a\left(\Phi_{\iota_{k}}-\Phi_{0}\right)\right|-\|a\|_{r}\left\|u_{k}\right\|_{r} \geq \\
\geq \frac{3}{4}\left|t_{k}\right|-\|a\|_{r} \varepsilon 2^{-k} \geq \frac{3}{4} \varepsilon^{\prime}-\frac{\varepsilon^{\prime}}{30} \geq \varepsilon^{\prime} / 4
\end{gathered}
$$

LEMMA 3.2. - There exists a continuous mapping $G \mapsto a_{G}$ from $W_{0}$ to $\left(C^{r-2}\right)^{*}=\mathcal{L}\left(C^{r-2}, \mathbf{R}\right)$ such that

$$
\left\|d T_{N}^{k}(G) u-\delta^{N k} a_{G}(u) v\right\|_{r-2} \leq C \delta^{N k} 2^{-k}\|u\|_{r-2}
$$

for all $G \in W_{0}$ and $u \in C^{r-2}, k=1,2, \ldots$, where $C$ is a constant. Moreover $a_{\Phi_{0}}=a$.

Proof. - Fix $G \in W_{0}$ and let $R_{k}=\delta^{-N k} d T_{N}^{k}(G) . R_{k}$ acts as a bounded linear operator on $C^{r-2}$. We have $R_{k+1} u=\delta^{-N} d T_{N}\left(G_{k}\right) R_{k} u$ where $G_{k}=T_{N}^{k} G$. For $\|u\|_{r-2} \leq 1$ we write $R_{k} u=\alpha_{k} v+\psi_{k}$ where $\alpha_{k} \in \mathbf{R}$ and $\psi_{k} \in C^{r-2}$ are linear on $u$, defined recursively as follows: $\alpha_{0}=0, \psi_{0}=u$ and if $\alpha_{k}$ and $\psi_{k}$ have been defined:

$$
\begin{gathered}
R_{k+1} u=\delta^{-N} d T_{N}\left(G_{k}\right)\left(\alpha_{k} v+\psi_{k}\right)= \\
=\alpha_{k} v+\alpha_{k} \delta^{-N}\left(d T_{N}\left(G_{k}\right)-\tilde{F}_{N}\right) v+\delta^{-N}\left(d T_{N}\left(G_{k}\right)-\tilde{F}_{N}\right) \psi_{k} \\
+a\left(\psi_{k}\right) v+\delta^{-N} Q_{N} \psi_{k}
\end{gathered}
$$


where $Q_{N} \psi_{k}=\tilde{F}_{N} \psi_{k}-\delta^{N} a\left(\psi_{k}\right) v$ and $\tilde{F}_{N}=d T_{N}\left(\Phi_{0}\right)$. Now $R_{k+1} u=$ $\alpha_{k+1} v+\psi_{k+1}$ where we define

$$
\begin{gathered}
\alpha_{k+1}=\alpha_{k}+a\left(\psi_{k}\right) \\
\psi_{k+1}=\alpha_{k} \delta^{-N}\left(d T_{N}\left(G_{k}\right)-\tilde{F}_{N}\right) v+\delta^{-N}\left(d T_{N}\left(G_{k}\right)-\tilde{F}_{N}\right) \psi_{k}+\delta^{-N} Q_{N} \psi_{k}
\end{gathered}
$$

Since $\left\|G_{k}-\Phi_{0}\right\|_{r}=O\left(2^{-k}\right)$ and the mapping from $C^{r}$ to $C^{r-2}$ given by $G \mapsto d T_{N}(G) \cdot v$ is differentiable at $\Phi_{0}$, we have that $\left\|\left(d T_{N}\left(G_{k}\right)-\tilde{F}_{N}\right) \cdot v\right\|_{r-2} \leq K 2^{-k}$ for some constant $K$ which can be taken independent of $G \in W_{0}$. If $\varepsilon$ and $\varepsilon^{\prime}$ are chosen small enough we have that the norm of the operator $d T_{N}\left(G_{k}\right)-\tilde{F}_{N}$ on $C^{r-2}$ is smaller that $\delta^{N} / 8$. By lemma 2.7 also the norm of $Q_{N}$ on $C^{r-2}$ is smaller than $\delta^{N} / 8$. Then

$$
\left\|\psi_{k+1}\right\|_{r-2} \leq \frac{1}{4}\left\|\psi_{k}\right\|_{r-2}+K \delta^{-N}\left|\alpha_{k}\right| 2^{-k}
$$

As $\alpha_{k+1}=\sum_{j=0}^{k} a\left(\psi_{j}\right)$, we obtain

$$
\left\|\psi_{k+1}\right\|_{r-2} \leq \frac{1}{4}\left\|\psi_{k}\right\|_{r-2}+2^{-k} K \delta^{-N}\|a\|_{r-2} \sum_{j=0}^{k-1}\left\|\psi_{j}\right\|_{r-2}
$$

Let $k_{0}$ be such that $K\|a\|_{r-2} \delta^{-N}\left(\frac{3}{2}\right)^{-k}<\frac{1}{8}$ for all $k \geq k_{0}$. Define $c_{1}$ a constant, independent of $G \in W_{0}$ and of $u$ in the unitary ball of $C^{r-2}$, such that $\left\|\psi_{k}\right\|_{r-2} \leq c_{1}\left(\frac{3}{4}\right)^{k}$ for $k=0,1, \ldots, k_{0}$. Then, by induction in $k$, it is easy to show that $\left\|\psi_{k}\right\|_{r-2} \leq c_{1}\left(\frac{3}{4}\right)^{k}$ for all $k \geq 0$. Therefore

$$
\left|\alpha_{k+1}-\alpha_{k}\right|=\left|a\left(\psi_{k}\right)\right|=O\left((3 / 4)^{k}\right)
$$

Thus $\alpha_{k}$ converges to a limit $a_{G}(u)$ linear on $u$. From (9) it is obtained that $\left\|\psi_{k}\right\|_{r-2}=O\left(2^{-k}\right)$. Therefore $\left|\alpha_{k}-a_{G}(u)\right|=O\left(2^{-k}\right)$ and we conclude

$$
\left\|R_{k} u-a_{G}(u) v\right\|_{r-2} \leq C 2^{-k}\|u\|_{r-2}
$$

for some constant $C$, for any $u \in C^{r-2}$ and any $G \in W_{0}$.

To show that $G \mapsto a_{G}$ is continuous, note first that the mapping $G \mapsto a \circ R_{k} \in\left(C^{r-2}\right)^{*}$ is continuous on $W_{0}$ for any $k$. For any $u \in C^{r-2}$ we have

$$
\left|a_{G}(u)-a\left(R_{k} u\right)\right|=\left|a\left(a_{G}(u) v-R_{k} u\right)\right| \leq C\|a\|_{r-2} 2^{-k}\|u\|_{r-2}
$$

so that $a \circ R_{k} \rightarrow a_{G}$ uniformly on $W_{0}$, whence the mapping $G \mapsto a_{G}$ is continuous. Note also that $a_{\Phi_{0}}$ is the projection $a$ because for $G=\Phi_{0}$ we have $R_{k} u \rightarrow a(u) \cdot v$. 
For later purposes we will need the following lemma that implies a convexity property for the $C^{r}$ norms:

LEMma 3.3. - There exists a constant $K$ such that for all $C^{2}$ map $w: D \mapsto \mathcal{L}_{r-2}\left(\mathbf{R}^{n}, \mathbf{R}^{n}\right)$

$$
\|D w\|_{0} \leq K\left(\|w\|_{2}\|w\|_{0}\right)^{1 / 2}
$$

Proof. - Let $c>0$ be a real number such that for any $P \in \mathcal{L}_{r-1}\left(\mathbf{R}^{n}, \mathbf{R}^{n}\right)$ determined by its $n^{r}$ real components $\left\{P_{j, \ldots, l, m}^{i}\right\}_{1 \leq i, j, \ldots, l, m \leq n}$

$$
\|P\| \leq c \max _{i, j, \ldots, l, m}\left|P_{j, \ldots, l, m}^{i}\right|
$$

Let $\gamma$ be the length of the smallest side of the parallelepiped $D$ and define

$$
K=\frac{2 c}{\gamma}+c \gamma
$$

Take $w: D \mapsto \mathcal{L}_{r-2}\left(\mathbf{R}^{n}, \mathbf{R}^{n}\right)$ of class $C^{2}$, and by contradiction suppose that for some $x_{0} \in D,\left\|D w\left(x_{0}\right)\right\|>K\left(\|w\|_{2}\|w\|_{0}\right)^{1 / 2}$. Thus, there exist $i, j, \ldots, l, m$ such that

$$
\left|\frac{\partial w_{j, \ldots, l}^{i}\left(x_{0}\right)}{\partial x_{m}}\right|>\frac{K}{c}\left(\|w\|_{2}\|w\|_{0}\right)^{1 / 2}
$$

As $\left\|D w(x)-D w\left(x_{0}\right)\right\| \leq\|w\|_{2}\left\|x-x_{0}\right\|$, we deduce

$$
\left|\frac{\partial w_{j, \ldots, l}^{i}(x)}{\partial x_{m}}-\frac{\partial w_{j, \ldots, l}^{i}\left(x_{0}\right)}{\partial x_{m}}\right| \leq\|w\|_{2} \eta
$$

for all $x \in D$ in a ball centered at $x_{0}$ at radius $\eta=\gamma \sqrt{\|w\|_{0} /\|w\|_{2}} \leq \gamma$. Therefore for such $x$ :

$$
\left|\frac{\partial w_{j, \ldots, l}^{i}(x)}{\partial x_{m}}\right|>\left(\frac{K}{c}-\gamma\right)\left(\|w\|_{2}\|w\|_{0}\right)^{1 / 2}=\frac{2}{\gamma}\left(\|w\|_{2}\|w\|_{0}\right)^{1 / 2}
$$

Integrating respect $x_{m}$ along a segment in $D$ with extremities $y_{1}$ and $y_{2}$ and length $\eta$

$$
\left|w_{j, \ldots, l}^{i}\left(y_{1}\right)-w_{j, \ldots, l}^{i}\left(y_{2}\right)\right|>\frac{2}{\gamma} \eta\left(\|w\|_{2}\|w\|_{0}\right)^{1 / 2}
$$

But the left member of the inequality above is smaller than $2\|w\|_{0}$ contradicting the definition of $\eta$. 
LEMMA 3.4. - There exists a constant $c_{0}$ such that if $G$ and $G_{1} \in$ $\left\{\Phi_{t}+w \in C^{r}:|t|<\varepsilon^{\prime},\|w\|_{r}<\varepsilon\right\}$ then

$$
\left\|T_{N} G_{1}-T_{N} G-d T_{N}(G) u\right\|_{r-2} \leq c_{0}\|u\|_{r-2}^{3 / 2}
$$

where $u=G_{1}-G$.

Proof. - In what follows $K$ stands for a constant that may vary in the different formulas. First we assert that

$$
\left\|T_{N}(G+u)-T_{N}(G)-d T_{N}(G) u\right\|_{r-2} \leq K \sum_{j=0}^{E\left(\frac{r-1}{2}\right)}\|u\|_{j}\|u\|_{r-j-1}
$$

where $E(x)$ denotes the greater integer smaller or equal than $x$.

We have that

$$
T_{N}(G+u)-T_{N}(G)-d T_{N}(G) u=\int_{0}^{1}\left(d T_{N}(G+t u)-d T_{N}(G)\right) \cdot u d t
$$

and so it is enough to prove that

$$
\left\|\left(d T_{N}(G+t u)-d T_{N}(G)\right) \cdot u\right\|_{r-2} \leq K \sum_{j=0}^{E\left(\frac{r-1}{2}\right)}\|u\|_{j}\|u\|_{r-j-1}
$$

for $t \in[0,1]$.

Computing explicitely $d T_{N}(G)$ as in the proof of the lemma 2.9 we see that $d T_{N}(G) \cdot u$ is the sum of compositions and multiplications of $u$ with maps depending on $G$ as follows:

$$
\begin{aligned}
d T_{N}(G) \cdot u & =\sum_{i} a_{i}(G) \cdot u\left(b_{i}(G)\right) \\
& +\sum_{i} c_{i}(G) \cdot\left(\prod_{j=1}^{s(i)} D G\left(e_{i, j}(G)\right)\right) \cdot u\left(h_{i}(G)\right)
\end{aligned}
$$

where $a_{i}(G), b_{i}(G), c_{i}(G), e_{i, j}(G), h_{i}(G)$ are maps in $C^{r}$ that depend non linearly on $G \in C^{r}$. The transformations $G \mapsto a_{i}(G)$, etc. have Fréchet functional derivatives respect to $G$ when looked from $C^{r}$ to $C^{r-2}$.

Thus

$\left(d T_{N}(G+t u)-d T_{N}(G)\right) \cdot u=A \cdot u+B \cdot u+C \cdot u+P \cdot u+E \cdot u+H \cdot u$ Vol. $15, n^{\circ} 3-1998$. 
where

$$
\begin{gathered}
A \cdot u=\sum_{i}\left(a_{i}(G+t u)-a_{i}(G)\right) \cdot u\left(b_{i}(G+t u)\right) \\
B \cdot u=\sum_{i} a_{i}(G) \cdot\left(u\left(b_{i}(G+t u)\right)-u\left(b_{i}(G)\right)\right. \\
C \cdot u=\sum_{i}\left(c_{i}(G+t u)-c_{i}(G)\right) \prod_{j} D(G+t u)\left(e_{i, j}(G+t u)\right) \cdot u\left(h_{i}(G+t u)\right) \\
P \cdot u=\sum_{i} c_{i}(G)\left\{\sum_{j=1}^{s(i)}\left(\prod_{k=1}^{j-1} D G\left(e_{i, k}(G+t u)\right)\right) \cdot t D u\left(e_{i, j}(G+t u)\right) \cdot\right. \\
\left.\cdot\left(\prod_{k=j+1}^{s(i)} D(G+t u)\left(e_{i, k}(G+t u)\right)\right)\right\} \cdot u\left(h_{i}(G+t u)\right) \\
E \cdot u=\sum_{i} c_{i}(G)\left\{\sum_{j=1}^{s(i)}\left(\prod_{k=1}^{j-1} D G\left(e_{i, k}(G)\right)\right)\right. \\
\cdot\left(\prod_{k=j+1}^{s(i)} D G\left(e_{i, k}(G+t u)\right)\right\} \cdot u\left(h_{i}(G+t u)\right) \\
H \cdot u=\sum_{i} c_{i}(G)\left(\prod_{j} D G\left(e_{i, j}(G)\right)\right) \cdot\left(u\left(h_{i}(G+t u)\right)-u\left(h_{i}(G)\right)\right)
\end{gathered}
$$

The hypothesis on $G$ and $G_{1}$ gives bounds for $\|G\|_{r}$ and $\|u\|_{r}$ and hence

$$
\begin{gathered}
\|A u\|_{r-2}=\left\|\sum_{i} \int_{0}^{1} d a_{i}(G+s t u) \cdot t u d s \cdot u\left(b_{i}(G+t u)\right)\right\|_{r-2} \\
\leq K \sum_{j=0}^{E\left(\frac{r-2}{2}\right)}\|u\|_{j}\|u\|_{r-j-2} \\
\|B u\|_{r-2}=\left\|\sum_{i} a_{i}(G) \int_{0}^{1} D u\left(b_{i}(G+s t u)\right) \cdot d b_{i}(G+s t u) \cdot t u d s\right\|_{r-2} \\
\leq K \sum_{j=0}^{E\left(\frac{r-1}{2}\right)}\|u\|_{j}\|u\|_{r-j-1}
\end{gathered}
$$


So $\|A \cdot u\|_{r-2}$ and $\|B \cdot u\|_{r-2}$ are bounded by the second term of the inequality (10). In a similar way are treated $C \cdot u, P \cdot u, E \cdot u$ and $H \cdot u$, obtaining the inequality (10).

From (10) and the lemma 3.3 the thesis is obtained: in fact, applying 3.3 to $w=D^{r-2} u$ we have

$$
\left\|D^{r-1} u\right\|_{0} \leq K\left(\|u\|_{r}\|u\|_{r-2}\right)^{1 / 2}
$$

As $\|u\|_{r}$ is bounded we obtain

$$
\|u\|_{r-1} \leq K\|u\|_{r-2}^{1 / 2}
$$

Substituting in (10) and using $\|u\|_{j} \leq\|u\|_{r-2}$ for $j=0,1, \ldots, r-2$ we obtain the inequality of the lemma.

By lemma 3.2 we can find $\varepsilon_{0}<\varepsilon$ such that if $G \in V_{0}=\left\{G \in W_{0}\right.$ : $\left.\left\|G-\Phi_{0}\right\|_{r}<\varepsilon_{0}\right\}$ then $a_{G}(v)>1 / 2$ and $\left\|a_{G}\right\|_{r-2} \leq 2\|a\|_{r-2}$.

LEMMA 3.5. - There exist positive numbers $\beta, \tau$ and $c$ such that, if $k$ is a sufficiently large positive integer and $G \in V_{0}, G_{1} \in W$ with $\left\|G_{1}-G\right\|_{r-2} \leq \beta \delta^{-N k}$ then

$$
\left\|T_{N}^{k} G_{1}-\Phi_{\delta^{N k} a_{G}\left(G_{1}-G\right)}\right\|_{r} \leq c \delta^{-N \tau k}
$$

Proof. - By lemma 3.4 there exists a constant $c_{1}>1$ such that if $G \in W_{0}$ then the norm of $d T_{N}^{k}(G)$ as an operator on $C^{r-2}$ is bounded by $c_{1} \delta^{N k}$ for any positive integer $k$.

Let

$$
\nu=\left(1-\delta^{-N / 2}\right)^{2} /\left(8 c_{0}^{2} c_{1}^{3}\right)
$$

where $c_{0}$ is as in the lemma 3.4, and let $c_{2}=\nu\left(1-\delta^{-N / 2}\right)$.

Let $\beta=\min \left(\nu, \zeta /\left(4\|a\|_{r-2}\right), c_{2} / C\right)$ with $C$ as in the lemma 3.2 and $\zeta$ as in the proposition 2.1.

Fix $G$ and $G_{1}$ satisfying the hypothesis and let $u=G_{1}-G$. Let $l_{0}$ be the first positive integer value of $l$ such that either $l>k$ or $\left|t_{l}\right|>\varepsilon^{\prime}$, with $T_{N}^{l} G_{1}=\Phi_{t_{l}}+u_{l}$.

We now define a sequence $\chi_{j}$ inductively by

$$
T_{N}^{l} G_{1}=T_{N}^{l} G+d T_{N}^{l}(G) u+\sum_{j=1}^{l} d T_{N}^{l-j}\left(T_{N}^{j} G\right) \chi_{j}
$$

Let us prove by induction that $\left\|\chi_{j}\right\|_{r-2} \leq c_{2} \delta^{3 N(j-k) / 2}$ for $j \leq l_{0}$ : 
First, for $j=1:\left\|\chi_{1}\right\|_{r-2}=\left\|T_{N} G_{1}-T_{N} G-d T_{N} G\left(G_{1}-G\right)\right\|_{r-2} \leq$ $c_{0}\left\|G_{1}-G\right\|_{r-2}^{3 / 2} \leq c_{0} \beta^{3 / 2} \delta^{-3 N k / 2} \leq c_{0} \nu^{3 / 2} \delta^{-3 N k / 2}=\nu \delta^{-3 N k / 2}(1-$ $\left.\delta^{-N / 2}\right) /\left(2 c_{1}\right)^{3 / 2} \leq \nu\left(1-\delta^{-N / 2}\right) \delta^{3 N(1-k) / 2}=c_{2} \delta^{3 N(1-k) / 2}$.

By induction, we suppose $\left\|\chi_{j}\right\|_{r-2} \leq c_{2} \delta^{3 N(j-k) / 2}$ holds for $j=$ $1, \ldots, l<l_{0}$. Applying (11) at $l+1$ and substituting $T_{N}^{l} G_{1}-T_{N}^{l} G$, we obtain:

$$
\chi_{l+1}=T_{N}^{l+1} G_{1}-T_{N}^{l+1} G-d T_{N}\left(T_{N}^{l} G\right)\left(T_{N}^{l} G_{1}-T_{N}^{l} G\right)
$$

By lemma 3.4 we have

$$
\left\|T_{N}^{l+1} G_{1}-T_{N}^{l+1} G-d T_{N}\left(T_{N}^{l} G\right)\left(T_{N}^{l} G_{1}-T_{N}^{l} G\right)\right\|_{r-2} \leq c_{0}\left\|T_{N}^{l} G_{1}-T_{N}^{l} G\right\|_{r-2}^{3 / 2}
$$

and so, for all $l<l_{0}$ :

$$
\begin{gathered}
\left\|\chi_{l+1}\right\|_{r-2} \leq c_{0}\left\|d T_{N}^{l}(G) u+\sum_{j=1}^{l} d T_{N}^{l-j}\left(T_{N}^{j} G\right) \chi_{j}\right\|_{r-2}^{3 / 2} \leq \\
\leq c_{0}\left(c_{1} \nu \delta^{N(l-k)}+c_{1} c_{2} \sum_{j=1}^{l} \delta^{N(l-j)} \delta^{3 N(j-k) / 2}\right)^{3 / 2} \leq \\
\leq c_{0}\left(c_{1} \nu \delta^{N(l-k)}+c_{1} c_{2} \delta^{3 N(l-k) / 2} /\left(1-\delta^{-N / 2}\right)\right)^{3 / 2}= \\
=c_{0}\left(c_{1} \nu \delta^{N(l-k)}+c_{1} \nu \delta^{3 N(l-k) / 2}\right)^{3 / 2} \leq c_{0}\left(2 c_{1} \nu \delta^{N(l-k)}\right)^{3 / 2}= \\
=c_{2} \delta^{3 N(l-k) / 2}<c_{2} \delta^{3 N(l+1-k) / 2}
\end{gathered}
$$

It now follows that, if $l \leq l_{0}$ then

$$
\begin{gathered}
\left\|T_{N}^{l} G_{1}-T_{N}^{l} G-d T_{N}^{l}(G) u\right\|_{r-2}=\left\|\sum_{j=1}^{l} d T_{N}^{l-j}\left(T_{N}^{l} G\right) \chi_{j}\right\|_{r-2} \leq \\
\leq \sum_{j=1}^{l} c_{1} \delta^{N(l-j)} c_{2} \delta^{3 N(j-k) / 2} \leq c_{1} c_{2} \delta^{3 N(l-k) / 2} /\left(1-\delta^{-N / 2}\right)=c_{1} \nu \delta^{3 N(l-k) / 2}
\end{gathered}
$$

By lemma 3.2

$$
\left\|d T_{N}^{l} G u-\delta^{l N} a_{G}(u) v\right\|_{r-2} \leq C \delta^{N l} 2^{-l}\|u\|_{r-2} \leq c_{2} 2^{-l} \delta^{N(l-k)}
$$


In what follows $K$ denotes a constant whose value may change in the different inequalities. Since $G \in W_{0}:\left\|T_{N}^{l} G-\Phi_{0}\right\|_{r} \leq K 2^{-l}$. Thus, for $l \leq l_{0}$ :

$$
\left\|T_{N}^{l} G_{1}-\Phi_{0}-\delta^{l N} a_{G}(u) v\right\|_{r-2} \leq K 2^{-l}+c_{1} \nu \delta^{3 N(l-k) / 2}
$$

Let $m$ be the nearest integer to $3 N k \log \delta /(2 \log 2+3 N \log \delta)$, therefore

$$
\frac{\delta^{-3 N / 4}}{\sqrt{2}} \leq \frac{2^{-m}}{\delta^{3 N(m-k) / 2}} \leq \sqrt{2} \delta^{3 N / 4}
$$

We now assert that $\left|a\left(T_{N}^{l} G_{1}-\Phi_{0}\right)\right|<\varepsilon^{\prime} / 4$, for $k$ large enough and $l \leq \min \left(l_{0}, m\right)$. In fact if $l \leq l_{0}$ we have

$$
\left\|T_{N}^{l} G_{1}-T_{N}^{l} G-\delta^{l N} a_{G}(u) v\right\|_{r-2} \leq c_{1} \nu \delta^{3 N(l-k) / 2}+c_{2} \delta^{N(l-k)}
$$

As

$$
\left\|\delta^{l N} a_{G}(u) v\right\|_{r-2} \leq K \delta^{N(l-k)}
$$

we deduce

$$
\left\|T_{N}^{l} G_{1}-T_{N}^{l} G\right\|_{r-2} \leq K \delta^{N(l-k)}
$$

and so

$$
\begin{aligned}
& \left|a\left(T_{N}^{l} G_{1}-\Phi_{0}\right)-a\left(T_{N}^{l} G-\Phi_{0}\right)\right| \leq K \delta^{N(l-k)} \leq K \delta^{N(m-k)} \\
& \quad \leq \delta^{-N((2 k \log 2) /(2 \log 2+3 N \log \delta)-1 / 2)}<\varepsilon^{\prime} / 8
\end{aligned}
$$

if $k$ is large enough.

To prove our assertion it is enough to show that $\left|a\left(T_{N}^{l} G-\Phi_{0}\right)\right|<\varepsilon^{\prime} / 8$. By proposition 2.1 we can write $T_{N}^{l} G=\Phi_{s_{l}}+u_{l}$. By (8) we have

$$
\begin{gathered}
\left|a\left(T_{N}^{l} G-\Phi_{0}\right)\right|=\left|a\left(\Phi_{s_{l}}-\Phi_{0}\right)+a\left(u_{l}\right)\right| \leq \frac{5}{4}\left|s_{l}\right|+\|a\|_{r}\left\|u_{l}\right\|_{r} \leq \\
\leq \frac{5}{2}\|a\|_{r} \varepsilon 2^{-l}+\|a\|_{r} \varepsilon 2^{-l} \leq \frac{7}{2} \frac{\varepsilon^{\prime}}{30}<\frac{\varepsilon^{\prime}}{8}
\end{gathered}
$$

Thus:

$$
\left|a\left(T_{N}^{l} G_{1}-\Phi_{0}\right)\right|<\frac{\varepsilon^{\prime}}{4} \text { for } l \leq \min \left\{l_{0}, m\right\}
$$

Using lemma 3.1, the definition of $l_{0}$ and the fact that $m<k$, we deduce that $\min \left\{l_{0}, m\right\}<l_{0}$. So, $m<l_{0}$. Applying the inequality (12):

$$
\left\|T_{N}^{m} G_{1}-\Phi_{0}-\delta^{m N} a_{G}(u) v\right\|_{r-2} \leq K \delta^{3 N(m-k) / 2}
$$

Vol. $15, n^{\circ} 3-1998$. 
and by proposition 2.1 we have for some $t$

$$
\left\|T_{N}^{m} G_{1}-\Phi_{t}\right\|_{r}<\varepsilon 2^{-m} \leq K \delta^{3 N(m-k) / 2}
$$

Writing $s_{0}=\delta^{m N} a_{G}(u)$ we deduce that

$$
\left\|\Phi_{t}-\Phi_{0}-s_{0} v\right\|_{r-2} \leq K \delta^{3 N(m-k) / 2}
$$

and so

$$
\begin{aligned}
\left|t-s_{0}\right| \leq K\left\|\Phi_{t}-\Phi_{s_{0}}\right\|_{r-2} & \leq K\left\|\Phi_{t}-\Phi_{0}-s_{0} v\right\|_{r-2}+K\left\|\Phi_{s_{0}}-\Phi_{0}-s_{0} v\right\|_{r-2} \leq \\
& \leq K \delta^{3 N(m-k) / 2}+K s_{0}^{2}
\end{aligned}
$$

As

$$
s_{0}^{2}=\left(\delta^{m N} a_{G}(u)\right)^{2} \leq\left(\delta^{m N}\left\|a_{G}\right\|_{r-2}\|u\|_{r-2}\right)^{2} \leq K \delta^{2 N(m-k)}
$$

we obtain $\left|t-s_{0}\right| \leq K \delta^{3 N(m-k) / 2}$ and $\left\|\Phi_{t}-\Phi_{s_{0}}\right\|_{r} \leq K \delta^{3 N(m-k) / 2}$. Using (13):

$$
\left\|T_{N}^{m} G_{1}-\Phi_{s_{0}}\right\|_{r} \leq K \delta^{3 N(m-k) / 2}
$$

By proposition 2.1 we can write, for $j=0,1, \ldots, k-m: T_{N}^{m+j} G_{1}=$ $\Phi_{s_{j}}+u_{j}$, with $s_{0}=\delta^{m N} a_{G}(u), u_{0}=T_{N}^{m} G_{1}-\Phi_{s_{0}}, s_{j+1}=\delta^{N}\left(s_{j}+a\left(u_{j}\right)\right)$ and

$$
\left\|u_{j}\right\|_{r} \leq 2^{-j}\left\|u_{0}\right\|_{r} \leq 2^{-j} K \delta^{3 N(m-k) / 2}
$$

We have $s_{k-m}=\delta^{N(k-m)} s_{0}+\sum_{j=0}^{k-m-1} \delta^{N(k-m-j)} a\left(u_{j}\right)$, from which we deduce that

$$
\left|s_{k-m}-\delta^{N k} a_{G}(u)\right| \leq \sum_{j=0}^{k-m-1} \delta^{N(k-m-j)} K 2^{-j} \delta^{3 N(m-k) / 2} \leq K \delta^{N(m-k) / 2}
$$

Thus $\left\|\Phi_{s_{k-m}}-\Phi_{\delta^{N k} a_{G}(u)}\right\|_{r} \leq K \delta^{N(m-k) / 2}$ and

$$
\begin{gathered}
\left\|T_{N}^{k} G_{1}-\Phi_{\delta^{N k} a_{G}(u)}\right\|_{r}=\left\|\Phi_{s_{k-m}}-\Phi_{\delta^{N k} a_{G}(u)}+u_{k-m}\right\|_{r} \\
\leq K \delta^{N(m-k) / 2}+K \delta^{3 N(m-k) / 2} \leq K \delta^{N(m-k) / 2}
\end{gathered}
$$

From the definition of $m$, we have that

$$
m-k \leq \frac{-2 k \log 2}{2 \log 2+3 N \log \delta}+\frac{1}{2}
$$

so $\left\|T_{N}^{k} G_{1}-\Phi_{\delta^{N k} a_{G}(u)}\right\|_{r} \leq c \delta^{-N \tau k}$ with $\tau=\log 2 /(2 \log 2+3 N \log \delta)$. 
THEOREM 3.6. - The local stable set of the renormalization $T_{N}$ is a $C^{1}$ submanifold of $C^{r}$ of codimension one. Its tangent subspace at $G_{0}$ is

$$
\left\{u \in C^{r}: a_{G_{0}}(u)=0\right\}
$$

where $a_{G_{0}}$ is defined in the lemma 3.2.

Proof. - Let us prove that the local stable set $V_{0}=\left\{G \in W_{0}\right.$ : $\left.\left\|G-\Phi_{0}\right\|_{r}<\varepsilon_{0}\right\}$ is a $C^{1}$ submanifold of codimension one.

Consider $G_{0} \in V_{0}$ and $G_{1}=G_{0}+t v$ for $t$ a small real number. By lemma 3.5:

$$
\left\|T_{N}^{k} G_{1}-\Phi_{s(t)}\right\|_{r} \leq c \delta^{-N \tau k}
$$

where $s(t)=\delta^{N k} t a_{G_{0}}(v)$, if $k$ is chosen so that $\beta /\left(\delta^{N}\|v\|_{r-2}\right) \leq \delta^{N k}|t| \leq$ $\beta /\|v\|_{r-2}$.

So $T_{N}^{k} G_{1}$ is in a small neighborhood of $\Phi_{s(t)}$ with

$$
\frac{\beta a_{G_{0}}(v)}{\delta^{N}\|v\|_{r-2}} \leq|s(t)| \leq \frac{\beta a_{G_{0}}(v)}{\|v\|_{r-2}}
$$

As $\Phi_{s(t)} \in W_{+}$if $t>0$ and $W_{+}$is an open set, we have that, for $t$ small enough and positive $T_{N}^{k} G_{1} \in W_{+}$. Therefore $G_{0}+t v \in W_{+}$if $t>0$ and small enough, say $0<t<\varepsilon^{\prime \prime}$. Analogously $G_{0}+t v \in W_{-}$if $0>t>-\varepsilon^{\prime \prime}$.

By continuity, if we fix $G_{0} \in V_{0}$ then for $G$ in a small neighborhood of $G_{0}$ the lines $\left\{G+t v, t \in\left(-\varepsilon^{\prime \prime}, \varepsilon^{\prime \prime}\right)\right\}$ have one and only one intersection with $V_{0}$. Let $H=\left\{u \in C^{r} ; a_{G_{0}}(u)=0\right\}$. It is a codimension one subspace of $C^{r}$. For $u \in H$ with $\|u\|_{r}$ sufficiently small, there exists an unique small real number $\chi(u)$ such that $G_{0}+u+\chi(u) v \in V_{0}$. We will show that $\chi$ is of class $C^{1}$ and $d \chi(0)=0$. Thus $V_{0}$ is locally diffeomorphic to $H$ and is a $C^{1}$ submanifold of codimension one.

$\chi$ is continuous: in fact if $u_{j} \rightarrow u$ with $G_{0}+u_{j}+\chi\left(u_{j}\right) v$ convergent, then it converges to a point in $V_{0}$ because $V_{0}$ is a relatively closed set, and so $\chi\left(u_{j}\right) \rightarrow \chi(u)$. Let us prove that $\chi$ is of class $C^{1}$. Let $G_{i}=G_{0}+u_{i}+\chi\left(u_{i}\right) v$ for $i=1,2$, so $G_{1}, G_{2} \in V_{0}$. Let $k$ be the largest integer number so that $\left\|G_{2}-G_{1}\right\|_{r-2} \leq \beta \delta^{-N k}$. Then $\beta \delta^{-N(k+1)}<\left\|G_{2}-G_{1}\right\|_{r-2} \leq\left\|G_{2}-G_{1}\right\|_{r}$ and by lemma 3.5

$$
\left\|T_{N}^{k} G_{2}-\Phi_{s}\right\|_{r} \leq c \delta^{-N \tau k}
$$

for $s=\delta^{N k} a_{G_{1}}\left(G_{2}-G_{1}\right)$. Also by lemma 3.5,

$$
\left\|T_{N}^{k} G_{2}-\Phi_{0}\right\|_{r} \leq c \delta^{-N \tau k}
$$

Vol. $15, n^{\circ} 3-1998$. 
Therefore

$$
|s| \leq K\left\|\Phi_{s}-\Phi_{0}\right\|_{r} \leq 2 c K \delta^{-N \tau k}
$$

for some constant $K$ and hence

$$
\left|a_{G_{1}}\left(G_{2}-G_{1}\right)\right| \leq 2 c K\left(\delta^{-N k}\right)^{\tau+1}=O\left(\left\|G_{2}-G_{1}\right\|_{r}^{\tau+1}\right)
$$

So:

$a_{G_{1}}\left(u_{2}-u_{1}\right)+a_{G_{1}}(v)\left(\chi\left(u_{2}\right)-\chi\left(u_{1}\right)\right)=O\left(\left\|u_{2}-u_{1}+\left(\chi\left(u_{2}\right)-\chi\left(u_{1}\right)\right) v\right\|_{r}^{\tau+1}\right)$

We assert that $\left|\chi\left(u_{2}\right)-\chi\left(u_{1}\right)\right|=O\left(\left\|u_{2}-u_{1}\right\|_{r}\right)$. In fact, by contradiction if it were sequences $\left\{u_{1, j}\right\}$ and $\left\{u_{2, j}\right\}$ such that $\left\|u_{1, j}-u_{2, j}\right\|_{r} \rightarrow 0$ and $\left\|u_{2, j}-u_{1, j}\right\|_{r}=\mathrm{o}\left(\chi\left(u_{2, j}\right)-\chi\left(u_{1, j}\right)\right)$, then

$$
a_{G_{1}}(v)\left(\chi\left(u_{2, j}\right)-\chi\left(u_{1, j}\right)\right)=O\left(\left|\chi\left(u_{2, j}\right)-\chi\left(u_{1, j}\right)\right|^{\tau+1}\right)
$$

which is absurd because $a_{G_{1}}(v) \neq 0$.

Then

$$
a_{G_{1}}(v)\left(\chi\left(u_{2}\right)-\chi\left(u_{1}\right)\right)+a_{G_{1}}\left(u_{2}-u_{1}\right)=O\left(\left\|u_{2}-u_{1}\right\|_{r}^{\tau+1}\right)
$$

and $\chi$ is differentiable at $u_{1}$ with $d \chi\left(u_{1}\right)=-\left(a_{G_{1}}(v)\right)^{-1} a_{G_{1}}$.

From the continuity of $G_{1} \mapsto a_{G_{1}}$ we deduce the continuity of $d \chi$ and hence $V_{0}$ is a $C^{1}$ submanifold in a neighborhood of $G_{0}$.

For $u \in C^{r}$ we have defined $J u=a(u) v$ the projection on the subspace $[v]=U$ of dimension one tangent to the unstable manifold $W^{u}=\left\{\Phi_{t}\right\}$ at $\Phi_{0}$. Thus $I-J$ is the projection on the subspace $S=\{\operatorname{ker} a\}$ tangent to the stable manifold $W^{s}$ at $\Phi_{0}$.

Let us consider the decomposition in $C^{r}: u=\left(u_{1}, u_{2}\right)$ where $u_{1}=J u \in$ $U$ and $u_{2}=u-u_{1} \in S$. In a neighborhood of $\Phi_{0}$ the unstable manifold $W^{u}$ is diffeomorphic to a neighborhood $B^{u}$ of 0 of its tangent space $U$. We have a $C^{1}$ map $\theta_{2}$ such that $\Phi_{0}+\left(u_{1}, \theta_{2}\left(u_{1}\right)\right) \in W^{u}$ for all $u_{1} \in B^{u} \subset U$. Moreover, $\theta_{2}(0)=0$ and $d \theta_{2}(0)=0$. Analogously, for the stable manifold we have a $C^{1}$ map $\theta_{1}$ such that $\Phi_{0}+\left(\theta_{1}\left(u_{2}\right), u_{2}\right) \in W^{s}$ for $u_{2} \in S$ in a neighborhood $B^{s}$ of 0 and $\theta_{1}(0)=0, d \theta_{1}(0)=0$.

We define the $C^{1}$ local change of coordinates $\theta$ from $B^{u} \times B^{s} \subset C^{r}$ to a neighborhood of $\Phi_{0}$ in $C^{r}$ :

$$
\theta\left(u_{1}, u_{2}\right)=\Phi_{0}+\left(u_{1}+\theta_{1}\left(u_{2}\right), u_{2}+\theta_{2}\left(u_{1}\right)\right)
$$

Thus $\theta$ is of class $C^{1}, \theta(0,0)=\Phi_{0}$, and $d \theta(0,0)$ is the identity. $\theta$ is a local $C^{1}$ diffeomorphism in $C^{r}$. Observe that $\theta$ transforms $B^{u} \subset U$ and $B^{s} \subset S$ 
onto respectively the local submanifolds $W^{u}$ and $W^{s}$. Now consider the expression of the transformation $T_{N}$ in the new coordinates:

$$
\tilde{T}_{N}=\theta^{-1} \circ T_{N} \circ \theta
$$

It has 0 as fixed point, $B^{u} \subset U$ and $B^{s} \subset S$ as local unstable and stable manifolds.

Increasing $r$ if necessary we can consider the local stable and unstable manifolds in $C^{r-1}$ which contain respectively those in $C^{r}$. As before, we can define the local diffeomorphism $\theta$ in $C^{r-1}$. The formal derivative $d T_{N}(G)$ for any $G \in C^{r}$ has been defined in the remark 2.10 as a bounded linear operator from $C^{r-1}$ to $C^{r-1}$. So we have $d \tilde{T}_{N}(u) \in \mathcal{L}\left(C^{r-1}, C^{r-1}\right)$, and $d T_{N}\left(\Phi_{0}\right)=d \tilde{T}_{N}(0)=\tilde{F}_{N}$. As $U$ and $S$ are invariant by $\tilde{F}_{N}$ we have

$$
\tilde{F}_{N}\left(u_{1}, u_{2}\right)=\left(A_{1} u_{1}, A_{2} u_{2}\right)
$$

where $A_{1} u_{1}=\delta^{N} u_{1}$ and by lemma 2.7

$$
\left\|A_{2} u_{2}\right\|_{r-1} \leq \frac{1}{2}\left\|u_{2}\right\|_{r-1}
$$

Let us write

$$
\tilde{T}_{N}\left(u_{1}, u_{2}\right)=\left(A_{1} u_{1}+\Delta_{1}\left(u_{1}, u_{2}\right), A_{2} u_{2}+\Delta_{2}\left(u_{1}, u_{2}\right)\right)
$$

We have $\Delta_{1}\left(0, u_{2}\right)=\Delta_{2}\left(u_{1}, 0\right)=0$ because $U$ and $S$ are invariant by $\tilde{T}_{N}$.

Considering the formal derivative of $\tilde{T}_{N}$ as a bounded linear operator from $C^{r-1}$ to $C^{r-1}$, we have bounded linear operators $d \Delta_{1}(u)$ and $d \Delta_{2}(u)$ on $C^{r-1}$. For $u$ in a neighborhood of $0,\left\|d \Delta_{1}(u)\right\|_{r-1}$ and $\left\|d \Delta_{2}(u)\right\|_{r-1}$ are smaller than a given positive number if $N$ is large enough (see the remark 2.10).

LEMMA 3.7 (The inclination lemma). - Given $\varepsilon>0$, there exists $N$ such that if $\left\{G_{\mu}\right\}$ is a $C^{1}$ curve of maps in $C^{r}$ intersecting transversally $W^{s}$ at $G_{0}$ and $D_{k}$ is the connected component through $T_{N}^{k} G_{0}$ of $\left\{T_{N}^{k} G_{\mu}\right\}$ in $B^{u} \times B^{s} \subset C^{r}$, then there exists $k_{0} \geq 0$ such that for $k \geq k_{0}, D_{k}$ is $C^{1}$ $\varepsilon$-close to $B^{u}$ in the space $C^{r-1}$.

Proof. - We have to repeat the same arguments of the proof of the inclination lemma of [14] for hyperbolic fixed points, using the $C^{r-1}$ norm of vectors in $C^{r}$, and the bounded operator $d \tilde{T}_{N}$ on $C^{r-1}$, with the bounds given above.

We recall the remark 1.8. Arguing in the space $C^{r-1}$ instead of $\mathcal{H}_{D}$ we deduce that the codimension one submanifolds $\Sigma_{m}$ are transversal to the 
unstable manifold $\left\{\Phi_{\mu}\right\}_{\mu<0}$ in $C^{r-1}$. Any curve of maps $\left\{G_{\mu}\right\} \subset C^{r-1}$ that has a transversal intersection with $\Sigma_{m}$ presents a period doubling bifurcation of period $2^{m}$ to $2^{m+1}$.

We also recall the definition of homoclinic bifurcation with unavoidable tangency (definition 1.9 and proposition 1.10). A version of the lemma 1.15 in the $C^{r}$ spacc can be stated with the same proof:

LEMMA 3.8. - There exist an interval $[a, b]$ with $b>a>0$ and open sets $N, N_{1}$ and $N_{2}$ in $C^{r}$ containing respectively $\left\{\Phi_{t} ; t \in[a, b]\right\}, \Phi_{a}$ and $\Phi_{b}$ such that any continuous arc $\left\{G_{\mu}\right\}$ in $N$ with extremities in $N_{1}$ and $N_{2}$ exhibits a homoclinic bifurcation with unavoidable tangency.

Taking preimages of $N_{1}, N_{2}$ and $N$ by a sufficiently large iterate of $T_{N}$, we can consider that they are as near $\Phi_{0}$ as wanted.

Proof of the theorem 2. - Let us take $\mathcal{W}$ the stable manifold $W^{s}$ of $T_{N}$. As $\left\{G_{\mu}\right\}$ intersects transversally $W^{s}$ at $G_{0}$ we have that $\left.\frac{\partial}{\partial \mu} G_{\mu}\right|_{\mu=0}$ is not contained in the tangent subspace $T_{G_{0}} W^{s}$, that is, by theorem 3.6 ,

$$
a_{G_{0}}\left(\left.\frac{\partial}{\partial \mu} G_{\mu}\right|_{\mu=0}\right)=h \neq 0
$$

Let us suppose $h>0$. As $\left\{G_{\mu}\right\}$ is differentiable respect $\mu$ : $\left\|G_{\mu}-G_{0}\right\|_{r-2} \leq$ $c_{3}|\mu|$ for some constant $c_{3}$ and for all $|\mu|$ small enough. For any large inleger $k>0$

$$
\left\|G_{\delta^{-N k} \mu}-G_{0}\right\|_{r-2} \leq c_{3}|\mu| \delta^{-N k} \leq \beta \delta^{-N k} \text { for all } \mu \in\left[-\frac{\beta}{c_{3}}, \frac{\beta}{c_{3}}\right]
$$

Thus, by lemma 3.5 :

$$
\left\|T_{N}^{k} G_{\delta^{-N k} \mu}-\Phi_{s(\mu)}\right\|_{r} \leq c \delta^{-N \tau k}
$$

for $s(\mu)=\delta^{N k} a_{G_{0}}\left(G_{\delta-N k}-G_{0}\right)$ for all $|\mu| \leq \beta / c_{3}$. Now

$$
s(\mu)=\delta^{N k} a_{G_{0}}\left(G_{\delta-N k}-G_{0}\right)=\delta^{N k} h \delta^{-N k} \mu+\mathrm{o}(\mu)=h \mu+\mathrm{o}(\mu)
$$

Thus $T_{N}^{k} G_{\delta^{-N k} \mu}$ converges when $k \rightarrow \infty$ to $\Phi_{h \mu+o(\mu)}$ uniformly in $\mu \in\left[-\beta / c_{3}, \beta / c_{3}\right]$. Using the inclination lemma 3.7, the arc

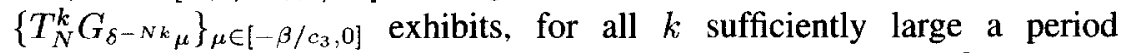
doubling bifurcation for a parameter value of $\mu \in\left[-\beta / c_{3}, 0\right]$. That is, there exists $\mu_{k} \in \delta^{-N k}\left[-\beta / c_{3}, 0\right] \rightarrow 0$ where $G_{\mu_{k}}$ presents a period doubling bifurcation.

Using the lemma 3.8 the arc $\left\{T_{N}^{k} G_{\delta-N k_{\mu}}\right\}_{\mu \in\left[0, \beta / c_{3}\right]}$ will have a subarc in $N$, with extremities in $N_{1}$ and $N_{2}$ and thus, it exhibits a homoclinic tangency for some value of $\mu \in\left[0, \beta / c_{3}\right]$. Therefore, there exists $\bar{\mu}_{k} \in \delta^{-N k}\left[0, \beta / c_{3}\right] \rightarrow 0$ such that $G_{\bar{\mu}_{k}}$ exhibits a homoclinic tangency. 


\section{REFERENCES}

[1] M. Benedicks and L. Carleson, The dynamics of the Hénon map, Annals of Math., Vol. 133, 1991, pp. 73-169.

[2] P. Collet, J. P. Eckmann and H. KoCH, Period doubling bifurcations for families of maps on $R^{n}$, J. Stat. Phys., Vol. 25, N.1, 1981, pp. 1-14.

[3] P. Coullet and C. Tresser, Itérations d'endomorphisms et groupe de rénormalisation, C.R. Acad. Sci. Paris, 287, 1978, pp. 577-588.

[4] A. M. Davie, Period doubling for $C^{2+\varepsilon}$ mappings, Preprint Univ. Edinburgh, 1992.

[5] J. P. Eckmann and P. WitTwer, 1987, A complete proof of the Feigenbaum conjectures, $J$. Stat. Physics, Vol. 46, N.3/4, 1987, pp. 455-475.

[6] M. J. Feigenbaum, Quantitative universality for a class of nonlinear transformations, J. Stat. Physics, Vol. 19, 1978, pp. 25-52

[7] M. J. Feigendaum, The universal metric properties of non-linear transformations, J. Stat. Physics, Vol. 21, 1979, pp. 669-706

[8] M. Hirsch, C. Pugh and M. Shub, Invariant manifolds, Lect. Notes in Math., Vol. 583, Springer.

[9] O. LANFORD III, A computer assisted proof of the Feigenbaum's conjectures, Bulletin of the A.M.S., Vol. 6, 1982 , pp. 427-434.

[10] J. C. Martín-Rivas, Homoclinic bifurcations and cascades of period doubling in higher dimensions, Tese do IMPA., Rio de Janeiro.

[11] L. MoRA and M. Viana, Abundance of Strange Attractors, Acta Math., Vol. 171, 1993, pp. $1-71$.

[12] S. Newhouse, The abundance of wild hyperbolic sets and nonsmooth stable sets for diffeomorphisms, Publ. Math. I.H.E.S. Vol. 50, 1979, pp. 101-151.

[13] S. Newhouse, J. Palis and F. Takens, Bifurcations and Stability of Families of Diffeomorphisms, Publ. Math. I.H.E.S, Vol. 57, 1983, pp. 5-71.

[14] J. Palis and W. De Melo, Geometric Theory of Dynamical Systems. An Introduction, Springer-Verlag, 1982, New York.

[15] J. Palis and F. TaKens, Hyperbolicity and the creation of homoclinic orbits, Annals of Math., Vol. 125, 1987, pp. 337-374.

[16] J. Palis and F. TAKens, Hyperbolicity and sensitive chaotic dynamics at homoclinic bifurcations, Cambridge University Press, 1993.

[17] J. Palis and M. VIANA, High dimension diffeomorphisms displaying infinitely many periodic attractors, Annals Math., Vol. 140, 1994, pp. 207-250.

[18] N. ROMERo, Persistence of homoclinic tangencies in higher dimensions, Tese do IMPA, Rio de Janeiro, 1992.

[19] F. Takens, Homoclinic Bifurcations, Proc. Int. Congress of Math., Berkeley, 1986, pp. 1229-1236.

[20] M. VIANA, Strange attractors in higher dimensions, Tese do IMPA, Rio de Janeiro, 1991.

[21] J. A. YORKE and K. T. ALLIGOOD, Cascades of period doubling bifurcations: a prerrequisite for horseshoes, Bull. A.M.S., Vol. 9, 1983, pp. 319-322.

(Manuscript received November 7, 1995.) 